\title{
I sistemi evoluzionistici nella ricerca della forma ideale
}

\author{
Leonardo Baglioni \\ Marco Fasolo \\ Matteo Flavio Mancini \\ Sofia Menconero
}

Abstract

Le ipotesi interpretative della forma originaria possono essere formulate e verificate attraverso l'uso di modelli geometrici ideali in grado di rappresentare in modo continuo le caratteristiche morfogenetiche dell'oggetto di studio. Dal punto di vista procedurale, a questa fase corrisponde la traduzione di un dato acquisito e descritto da un linguaggio discreto a uno interpretativo rappresentato per mezzo di un linguaggio continuo.

II presente studio vuole proporre un'indagine sulle potenzialità dei metodi computazionali di tipo evoluzionistico (Evolutionary Solver o Genetic Algorithm) applicati all'interpretazione del rilevamento e alla ricerca della forma ideale.

Tali algoritmi, impiegati per conoscere le caratteristiche morfogenetiche di un lacerto di affresco attribuito a Raffaello, hanno permesso non solo di ipotizzare la misura del raggio della curvatura longitudinale, già visibile da un'osservazione diretta, ma anche di mettere in evidenza una seconda curvatura, orizzontale e difficilmente percepibile a occhio nudo.

Parole chiave

sistemi evoluzionistici, geometria descrittiva, metodologie di rilievo per immagini, affresco, Raffaello.

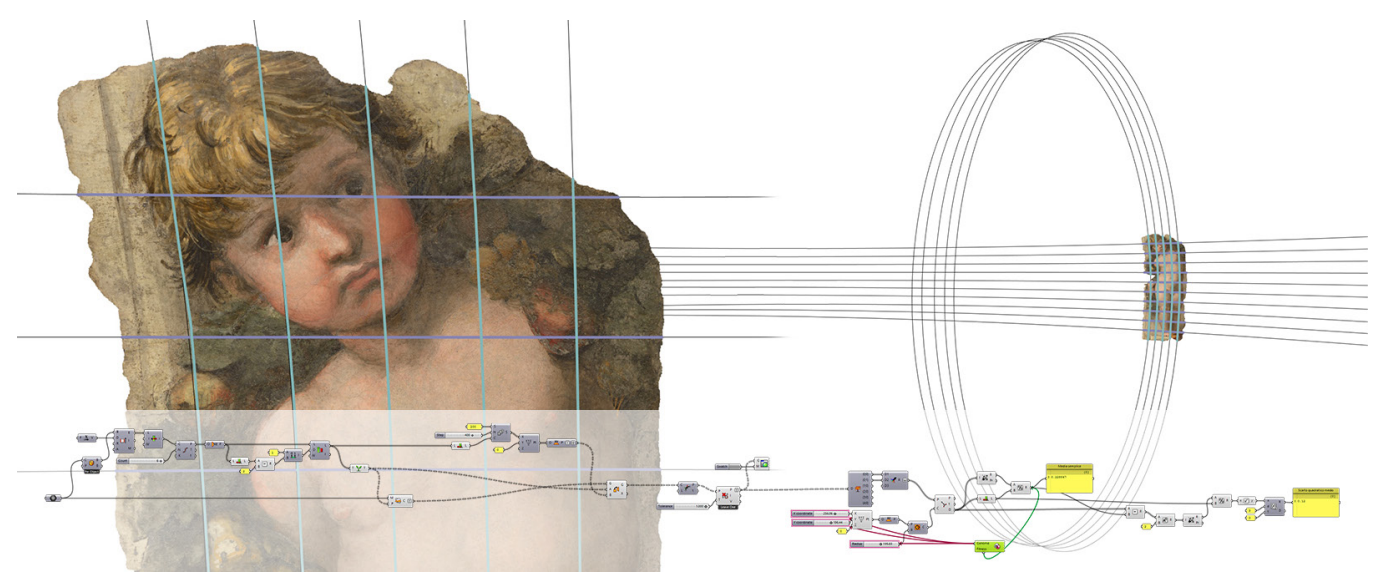




\section{Inquadramento}

Il rilevamento inteso come operazione di conoscenza volta alla comprensione profonda di un bene definisce un percorso articolato secondo diverse fasi e procedure coordinate e integrate tra loro in funzione della natura dell'oggetto e delle finalità dello studio. L'intero processo è orientato alla definizione di un modello conoscitivo in grado di rappresentare le evoluzioni e le metamorfosi che hanno interessato il bene nel tempo, a partire dalla sua idea iniziale di progetto fino ad arrivare allo stato di conservazione attuale. Grazie agli avanzamenti tecnologici degli ultimi anni, la fase di misurazione è diventata un'operazione di acquisizione massiva finalizzata soprattutto alla documentazione della superficie dell'oggetto. Gli effetti di questo approccio sono quelli di aver trasformato, in alcuni casi, il rilevamento in un'attività di carattere tecnico e tecnologico piuttosto che critico e interpretativo.

Le ipotesi interpretative della forma originaria possono essere formulate e verificate solo attraverso l'uso di modelli geometrici ideali in grado di rappresentare in modo continuo le caratteristiche morfogenetiche del bene. Si tratta di un passaggio di non facile soluzione in ragione di diversi aspetti: da un lato la natura dell'oggetto e delle vicissitudini che lo hanno coinvolto, dall'altro il carattere interdisciplinare e interpretativo dell'operazione. Dal punto di vista procedurale a questa fase corrisponde la trasformazione di un dato acquisito e descritto con un 'linguaggio discreto' a uno interpretativo rappresentato per mezzo di un 'linguaggio continuo'. II presente studio si inserisce all'interno di questo contesto particolarmente importante per restituire al rilevamento la dignità di attività di conoscenza cui abbiamo fatto riferimento, proponendo un'indagine delle potenzialità dei metodi computazionali di tipo evoluzionistico indirizzati alla ricerca della forma ideale.

Questi metodi sono stati introdotti a partire dai primi anni '60 ma solo recentemente hanno trovato larga diffusione grazie allo sviluppo di piattaforme aperte che ne consentono l'uso anche da parte di un pubblico di non programmatori. I sistemi Evolutionary Solver o Genetic Algorithm nei quali le logiche evoluzionistiche sono indirizzate alla soluzione di specifici problemi, sono caratterizzati dall'estrema flessibilità con la quale possono essere applicati in un'ampia varietà di problemi [Cheng 20 I 8; Slowik 2020]. II processo di calcolo si basa su di una modellizzazione nella quale le variabili sono rappresentate dai geni che caratterizzano i singoli individui (chiamati genomi) che popolano il fitness landscape (il modello multidimensionale nel quale si svolgerà il calcolo): variando i geni, cambierà lo stato del modello e di conseguenza cambierà, in meglio o in peggio in funzione degli obiettivi del calcolo, l'idoneità finale (fig. I).

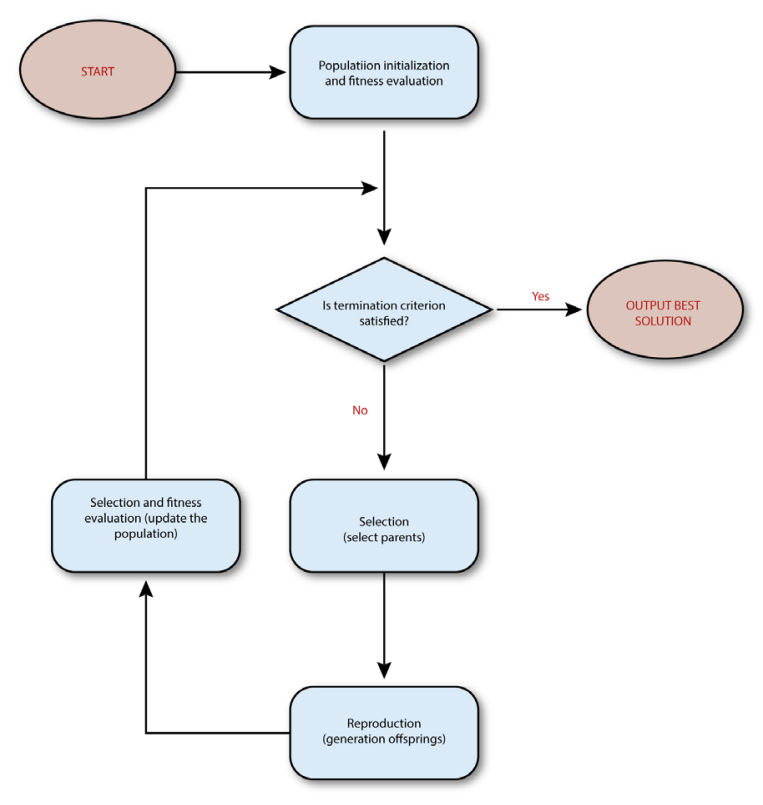


Ma qual è il risultato migliore? La risposta a questa domanda apparentemente banale definisce una caratteristica fondamentale degli 'algoritmi evoluzionistici': questi metodi computazionali, infatti non garantiscono un'unica soluzione. Fino a che non viene specificato il valore di riferimento su ciò che può essere considerato 'sufficientemente adatto' il processo verrebbe ripetuto all'infinito senza mai raggiungere una risposta esatta, peraltro senza avere la possibilità di riconoscerla.

Nonostante questo limite superabile solo con una serie di scelte definite dall'utente che restituiscono un carattere critico all'intera operazione, gli ‘algoritmi evoluzionistici' possono offrire contributi notevoli nella fase interpretativa di una forma rilevata.

Un caso applicativo sul quale collaudare questa metodologia ci è stato offerto dalla collaborazione con l'Accademia Nazionale di San Luca, la quale ci ha messo a disposizione un bene di sua proprietà per effettuare su di esso alcune indagini geometriche. II bene in questione è il Putto reggifestone attribuito a Raffaello che venne donato alla San Luca nel I834, tramite lascito testamentario, da Jean Baptiste Wicar figura di spicco dell'Accademia [I].

Si tratta di un frammento di dipinto su intonaco di circa 40× $107 \mathrm{~cm}$ aderente a un supporto in masonite collegato a una tavoletta mediante delle staffe in ferro. La superficie del lacerto appare evidentemente curva nel senso longitudinale mentre una seconda curvatura, non visibile a occhio nudo, interessa la sezione orizzontale come avremo modo di dimostrare.

\section{Dalla forma reale alla forma ideale}

Data la natura dell'oggetto di indagine e le finalità di studio mirate alla conoscenza morfologica del lacerto di affresco, la sua acquisizione è stata progettata attribuendo delle priorità in termini di controllo geometrico e cromatico. In quest'ottica, e tenendo in considerazione le dimensioni dell'oggetto non particolarmente ingenti, la tecnica fotogrammetrica ha permesso di raggiungere entrambi gli obiettivi secondo le modalità espresse di seguito.

Innanzitutto, dovendo poi compiere degli studi di carattere morfogenetico, era imprescindibile avere dei dati accurati di rilievo geometrico, sia per quanto riguarda la corrispondenza formale generale, sia la risoluzione della micromorfologia.
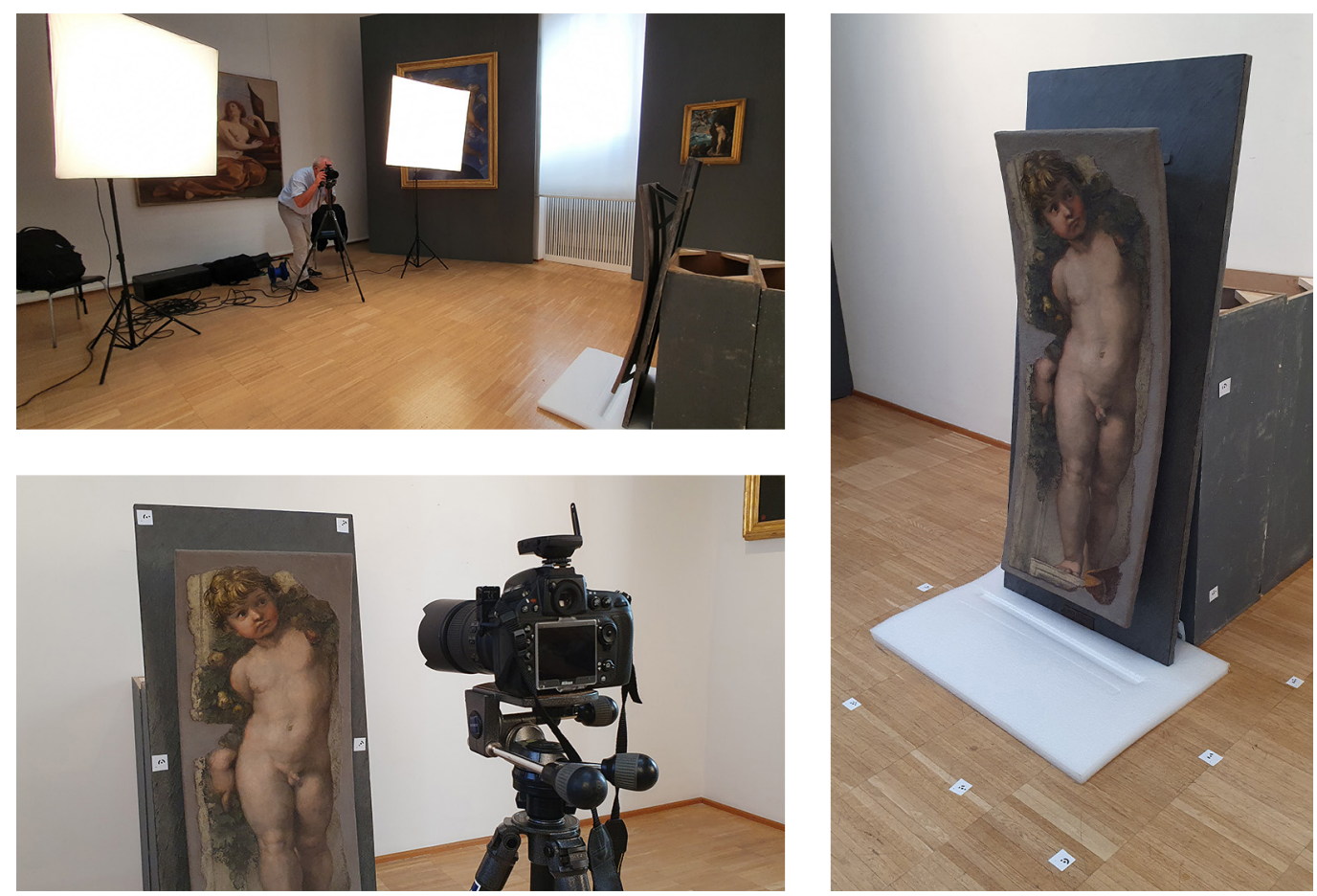
Fig. 3. Nuvola sparsa ottenuta in fase di allineamento che mostra anche la posizione dei punti di controllo e le distanze reciproche misurate.
Per raggiungere questo doppio obiettivo, l'acquisizione fotografica ha previsto diverse sessioni: una serie di scatti d'insieme con lente da $50 \mathrm{~mm}$ per riprendere l'oggetto per intero e i target, con lo scopo di assicurare una solida struttura geometrica all'allineamento delle successive fotografie; altri scatti più ravvicinati con la medesima lente finalizzati alla descrizione di dettaglio della micromorfologia; una serie di scatti con lente da $105 \mathrm{~mm}$ per rilevare il dettaglio figurativo ad alta risoluzione (fig. 2).

L'elaborazione dei dati fotografici è stata supportata metricamente dall'inserimento di 18 punti di controllo (target) posizionati in modo distribuito sulle superfici contigue al lacerto e di cui si sono rilevate le distanze con misurazione diretta. Delle 20 totali misurazioni condotte, 12 sono state utilizzate per scalare il modello e le restanti 8 come misure di controllo (fig. 3). La nuvola di punti, pulita e ritagliata a isolare i limiti del lacerto, conta 3.324 .005 punti e da questa è stata ricavata una mesh da 2.000.000 di poligoni (fig. 4).
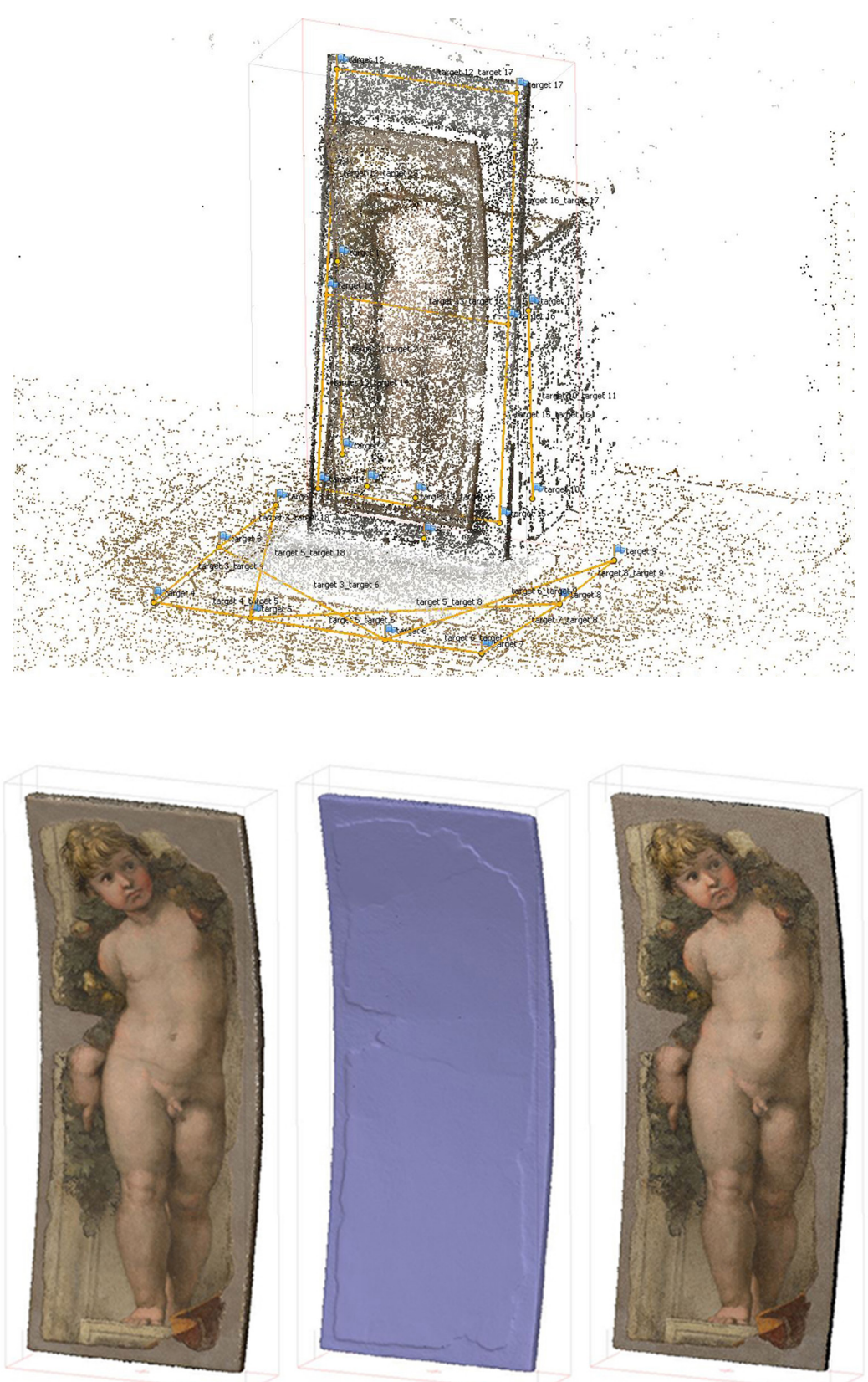
Fig. 5. Dettaglio della parte bassa del modello mesh che mostra lo stacco tra due diverse giornate di lavoro.
II controllo geometrico dei risultati, cui si mirava, è stato garantito da un errore di riproiezione sui singoli target molto contenuto (scarto medio pari a $0,17 \mathrm{~mm}$ ) e da un elevato dettaglio della micromorfologia della superficie mesh dichiarato dalla registrazione dello stacco tra due giornate di lavoro (fig. 5).

Data la peculiarità artistica dell'oggetto, parallelamente al controllo geometrico si è voluta garantire una resa cromatica altrettanto di qualità.

L'illuminazione, diffusa e controllata, insieme alla procedura di bilanciamento del bianco e agli scatti di dettaglio con obiettivo da $105 \mathrm{~mm}$ (migliore per risoluzione e nitidezza), hanno permesso di ottenere una texture da $8.192 \times 8.192$ pixel talmente definita da poter osservare i segni di spolvero (fig. 6).
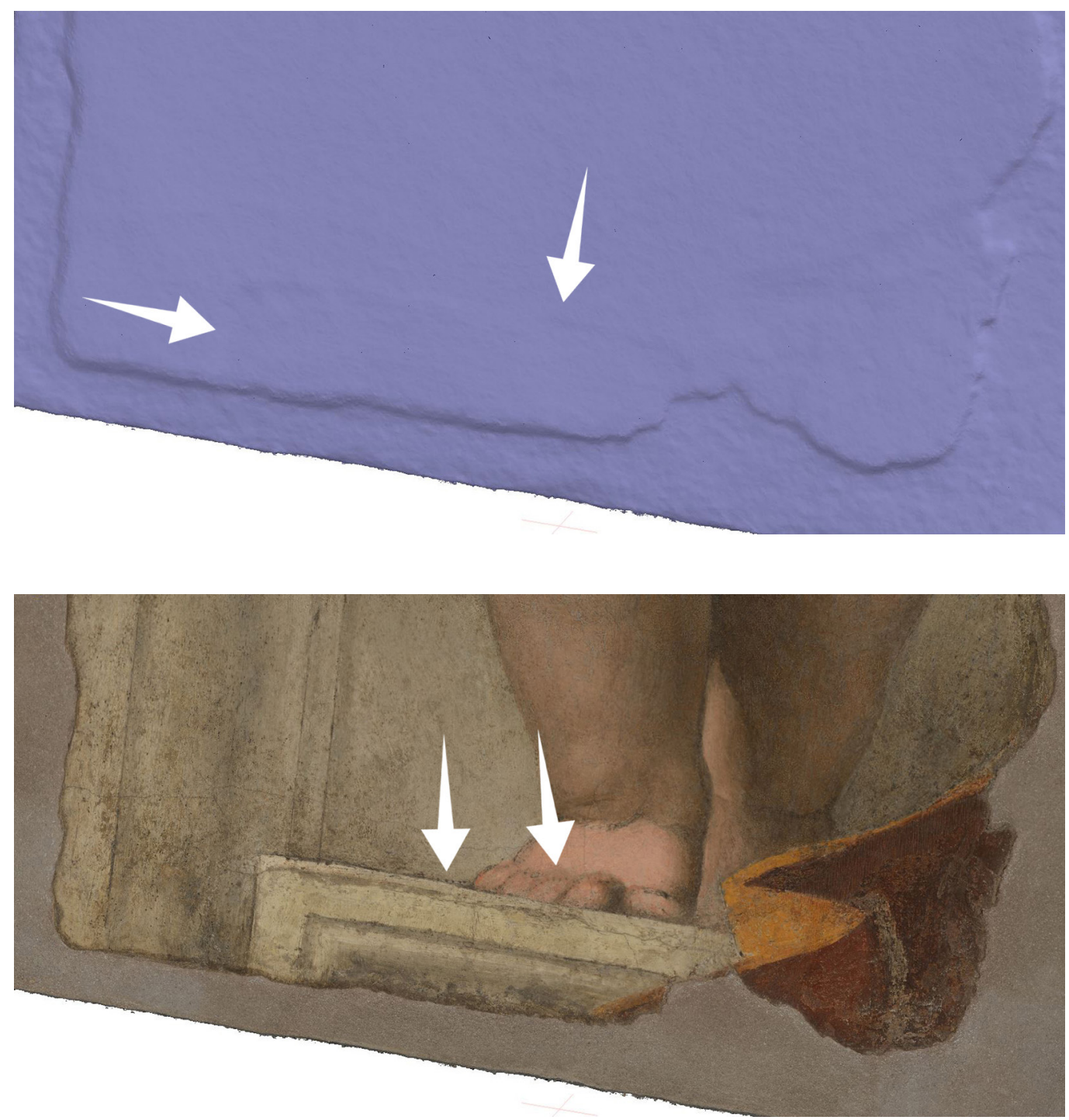

II dato ottenuto dal rilievo fotogrammetrico è una mesh, una superficie definita da una struttura poliedrica discreta. Lo studio delle caratteristiche geometriche è stato condotto attraverso l'analisi di sezioni campione verticali e orizzontali generate attraverso l'intersezione con due fasci di piani paralleli, che hanno restituito: cinque sezioni verticali (A-E) e dieci sezioni orizzontali (F-Q) (fig. 7). Ogni sezione è rappresentata da una linea spezzata (polilinea) i cui vertici sono stati campionati alla distanza di I cm. 
Fig. 7. Le cinque sezioni verticali (A-E) sezioni orizzontali (F-Q) considerate, e le rispettive
circonferenze calcolate.

Fig. 8 . I blocchi funzionali dell'algoritmo:

(a) generazione delle

sezioni; (b) ribaltamento delle sezioni sul piano di costruzione $x y$;

(c) campionamento

dei vertici delle sezion

(d) individuazione delle circonferenze di miglior approssimazione delle sezioni; (e) calcolo dello scarto quadratico medio.

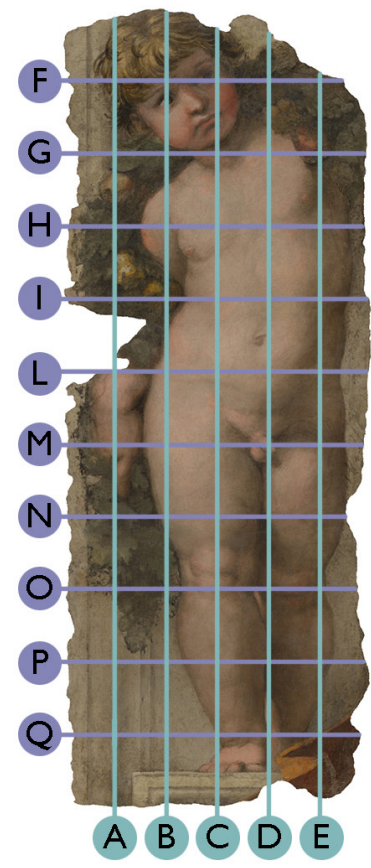

OOOP

A B C D E
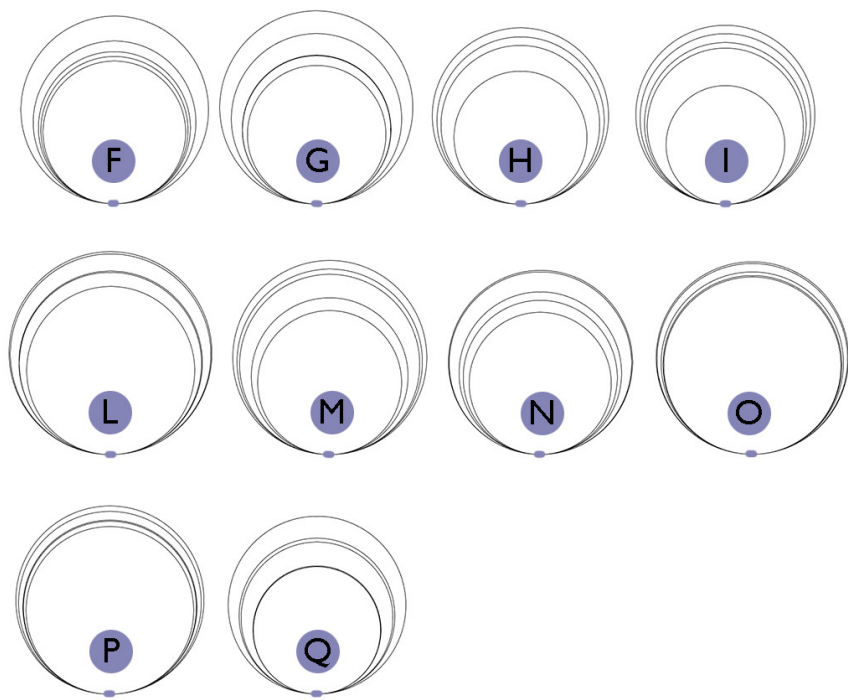

Per determinare il raggio di curvatura di un insieme discreto di punti è necessario scegliere un modello geometrico di riferimento che, nel caso in questione, è stato quello della circonferenza. Questa risulta infatti una delle curve generatrici più comuni nell'architettura storica e, una volta definita come la circonferenza di miglior approssimazione dei punti dati, restituisce univocamente la proprietà ricercata attraverso il valore del suo raggio.

In questa sperimentazione si è fatto uso di una procedura algoritmica, programmata attraverso strumenti di Visual Programming Language (VPL) per gestire tutta l'elaborazione [2] (fig. 8). La definizione delle circonferenze di migliore approssimazione ha adottato un approccio del tipo generate-and-test, ovvero un criterio evoluzionistico, in cui la generazione delle soluzioni avviene in modo pressoché casuale per essere sottoposta solo in un secondo momento a una rigorosa selezione critica [Mitchell 1990, pp. 179, 180].

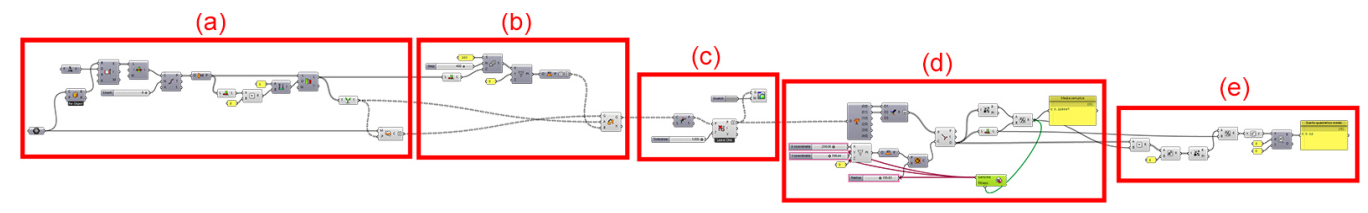

Questo procedimento euristico è sempre più applicato, sfrutta le potenzialità di calcolo oggi disponibili per simulare digitalmente una popolazione di prototipi all'interno dei quali andare a cercare la soluzione ottimale o, per meglio dire, la migliore disponibile in un dato numero di iterazioni, secondo il paradigma del Search don't sort che pervade sempre più la seconda svolta digitale dei Big Data [Carpo 20 17, pp. 23-55].

Ogni circonferenza è caratterizzata da un genoma $(x, y, R)$ definito dalle coordinate cartesiane del centro e dal valore del raggio. L'algoritmo evoluzionistico ricerca la terna corrispondente alla circonferenza di miglior approssimazione dei punti analizzati, cioè quella circonferenza che riduce al minimo la somma delle distanze dai vertici della polilinea sezione. Per impostare e indirizzare il processo evoluzionistico si devono compiere alcune scelte cri- 
tiche: decidere il numero di individui di ciascuna generazione (I00); indicare la percentuale di individui candidata a generare la successiva generazione (I0\%); stabilire la percentuale di endogamia, ovvero se consentire l'accoppiamento di individui con genomi simili o favorire l'accoppiamento tra genomi diversi (-75\%); stabilire dopo quante occorrenze di uno stesso genoma questo venga scelto come il più soddisfacente e terminare il calcolo ( I00).

La percentuale di endogamia degli accoppiamenti è particolarmente importante: nel caso in cui questa condizione sia consentita si otterrà una più rapida convergenza verso un risultato ma si aumenterà il rischio di ottenere un falso positivo nel caso di problemi con più possibili soluzioni; all'opposto, qualora sia impedita l'endogamia, la convergenza sarà più lenta ma la maggiore variabilità del genoma comporterà una minore probabilità di falsi positivi (fig. 9).

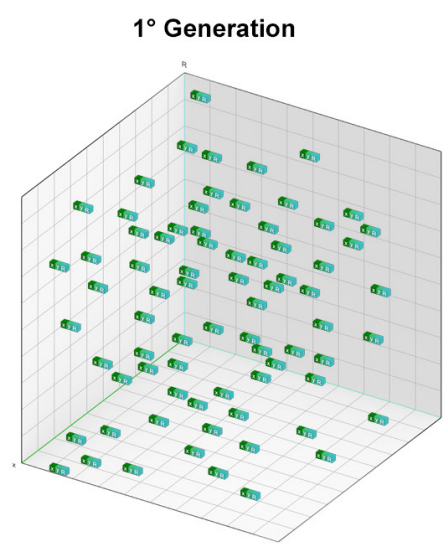

Classifing genomas based on their fitness

1. $(x, y, R)$

2. $(x, y, R)^{2}$

3. $(x, y, R)_{3}$

4. $(x, y, R)_{4}$

5. $(x, y, R)_{5}$

6. $(x, y, R)_{6}$

7. $(x, y, R)_{7}$

8. $(x, y, R)_{8}$

9. $(x, y, R)^{9}$

10. $(x, y, R)$

...

n. $(x, y, R)_{n}$ $2^{\circ}$ Generation

High imbreeding

$(x, y, R)_{1}+(x, y, R)_{2}$

$(x, y, R)_{1}+(x, y, R)_{3}$

$(x, y, R)_{3}+(x, y, R)_{4}$

...

Imbreeding

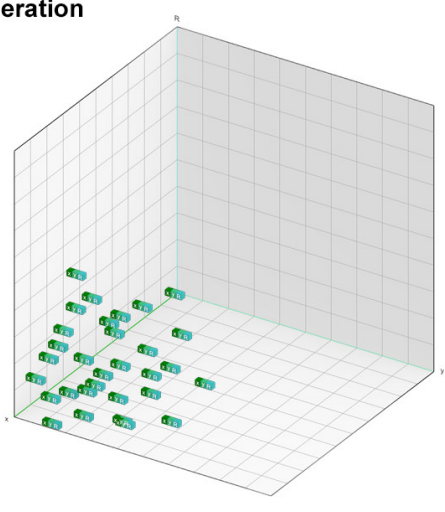

Low imbreeding

(0\% -> - 100\%)

$(x, y, R)_{1}+(x, y, R)_{10}$

$(x, y, R)_{1}+(x, y, R)_{8}$

$(x, y, R)_{2}+(x, y, R)_{9}$
Fig. 9. Creazione di successive generazioni in un algoritmo evoluzionistico.

I risultati forniti dagli 'algoritmi evoluzionistici' non sono univoci, possono evidenziare oscillazioni tra ripetizioni successive, pertanto ogni sezione è stata testata cinque volte e, per ogni reiterazione, sono state calcolate la media delle distanze tra la circonferenza e i vertici della polilinea e il loro scarto quadratico medio (deviazione standard). Quest'ultimo consente di valutare la distribuzione delle distanze intorno al valore medio e quindi la consistenza del risultato (figg. 10, I I). Per ciascuna sezione è stata poi calcolata la media dei raggi delle cinque circonferenze individuate e il loro scarto quadratico medio.

Le sezioni verticali (A-E) hanno restituito risultati precisi e accurati, fornendo un'indicazione attendibile riguardo il raggio di curvatura verticale medio che risulta di $195,978 \mathrm{~cm}$, con uno scarto quadratico medio di $2,973 \mathrm{~cm}$, pari a I,52\% del raggio medio (tab. I).

La generazione delle sezioni orizzontali ( $F-Q$ ) ha svelato l'esistenza di una seconda curvatura, difficilmente percepibile a un'osservazione diretta, che è stata pertanto oggetto della stessa analisi effettuata per le sezioni verticali.

In quest'ultimo caso i risultati presentano notevoli oscillazioni già nelle singole letture e ciò si propaga negativamente sul raggio di curvatura media orizzontale che risulta di $677,417 \mathrm{~cm}$ ma con uno scarto quadratico medio di $51,531 \mathrm{~cm}$, pari al 7,61\% del raggio medio, valore 
Fig. 10. Sezione verticale $A$ e un dettaglio dell'approssimazione (raggio medio 196.346 $\mathrm{cm}$; scarto quadratico medio $0.737 \mathrm{~cm}$ ).

Fig. I I. Sezione orizzontale L e un dettaglio dell'approssimazione (raggio medio 763.500 $\mathrm{cm}$; scarto quadratico medio $52.438 \mathrm{~cm}$ ). significativamente elevato (tab. 2). Questo risultato può essere ricondotto alla natura del dato analizzato che è caratterizzato sia da un esiguo valore di curvatura sia da una ridotta estensione della dimensione orizzontale del lacerto.

I risultati che abbiamo descritto sono stati verificati analizzando i medesimi dati con algoritmi di analisi statistica basati sulla migliore approssimazione ai minimi quadrati (best fitting), utilizzando come modello l'equazione della circonferenza. Gli esiti sono, di fatto, coincidenti essendo trascurabili le differenze tra gli scarti. Pertanto, sono stati preferiti i risultati ottenuti attraverso ilVPL per la migliore ottimizzazione degli scarti restituiti e perché questa tecnica consente un migliore controllo dell'intero processo.
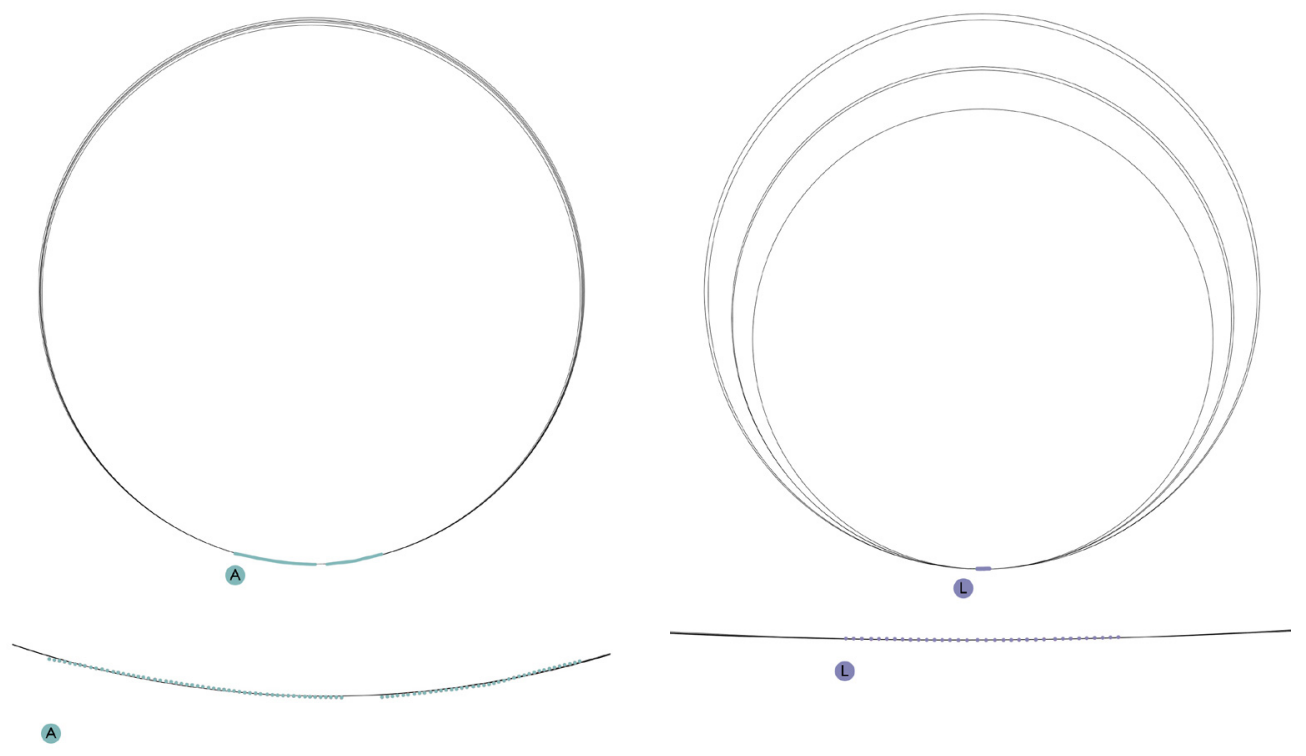

\section{Conclusioni}

La sperimentazione ha dimostrato le potenzialità degli algoritmi evoluzionistici come strumenti di analisi geometrica indirizzati all'interpretazione della forma. Le analisi condotte sul rilievo del lacerto hanno evidenziato la presenza di una seconda curvatura orizzontale che accompagna la prima, verticale e più evidente. II risultato raggiunto porta a non escludere la possibilità che il lacerto si possa considerare una porzione di superficie a doppia curvatura o, per meglio dire, a curvatura gaussiana positiva.

L'applicazione di questi algoritmi al problema della traduzione da un linguaggio di modellazione discreto a uno continuo non va intesa come puramente automatizzata. Sia la scelta del modello geometrico di riferimento sia le scelte in fase di impostazione delle norme che regolano la creazione delle generazioni, richiedono infatti conoscenze scientifiche specialistiche e la capacità di compiere scelte critiche tipiche delle fasi interpretative. La scelta del modello geometrico di riferimento è infatti un'operazione tipica della geometria differenziale, che studia una forma in relazione ad altre più semplici e dalle proprietà note.

Le potenzialità emerse nella sperimentazione aprono a possibili sviluppi sia verso l'uso di primitive geometriche bidimensionali sia all'applicazione in settori come quelli delle ricomposizioni archeologiche [Eslami et al. 2020] e architettoniche che vengono più spesso attuate attraverso altre tecniche analogiche e digitali.

\section{Note}

[I] Per le vicende storiche del Putto reggifestone, quali la sua autenticità, l'attribuzione e la provenienza originaria si rimanda a puntuali studi [Agostini 1983; Carloni 2008; Cellini 1960; Giacomini 2014; Ginzburg 2020; Salerno 1960; Ventra 2017].

[2] È stato utilizzato il linguaggio di programmazione visuale Grasshopper, implementato nel software di modellazione tridimensionale McNeel Rhinoceros 6.0 e il suo motore di calcolo evolutivo Galapagos. 
Tab. I.I raggi di curvatura delle sezion verticali ottenuti

attraverso l'algoritmo evoluzionistico.

\section{Ringraziament}

Si ringrazia l'Accademia Nazionale di San Luca per aver permesso i rilievi del Putto reggifestone e il prof. Riccardo Migliari per aver consigliato e supervisionato la sperimentazione.

Pur condividendo l'impianto metodologico e le conclusioni del lavoro, l'inquadramento del lavoro è da attribuire a Leonardo Baglioni e Marco Fasolo, mentre Sofia Menconero ha curato gli aspetti relativi al rilievo fotogrammetrico e Matteo Flavio Mancini quelli relativi all'analisi geometrica del lacerto.

\begin{tabular}{|c|c|c|c|c|c|}
\hline \multicolumn{6}{|c|}{ Sezioni verticali } \\
\hline Sezione & Raggio $[\mathrm{cm}]$ & Scarto medio $[\mathrm{cm}]$ & Scarto Quadratico Medio [cm] & Media raggi $[\mathrm{cm}]$ & Scarto Quadratico Medio [cm] \\
\hline A & 195,830 & 0,228 & 0,120 & \multirow{5}{*}{196,346} & \multirow{5}{*}{0,737} \\
\hline & 196,740 & 0,225 & 0,124 & & \\
\hline & 197,470 & 0,232 & 0,117 & & \\
\hline & 196,360 & 0,227 & 0,117 & & \\
\hline & 195,330 & 0,231 & 0,128 & & \\
\hline B & 194,100 & 0,227 & 0,118 & \multirow{5}{*}{194,190} & \multirow{5}{*}{1,329} \\
\hline & 193,750 & 0,230 & 0,118 & & \\
\hline & 196,690 & 0,241 & 0,127 & & \\
\hline & 193,670 & 0,228 & 0,120 & & \\
\hline & 192,740 & 0,226 & 0,119 & & \\
\hline $\mathrm{C}$ & 197,080 & 0,204 & 0,115 & \multirow{5}{*}{196,456} & \multirow{5}{*}{1,157} \\
\hline & 196,490 & 0,208 & 0,113 & & \\
\hline & 195,500 & 0,201 & 0,113 & & \\
\hline & 194,970 & 0,203 & 0,113 & & \\
\hline & 198,240 & 0,212 & 0,114 & & \\
\hline $\bar{D}$ & 190,750 & 0,154 & 0,098 & \multirow{5}{*}{191,968} & \multirow{5}{*}{1,598} \\
\hline & 193,920 & 0,170 & 0,095 & & \\
\hline & 193,430 & 0,165 & 0,095 & & \\
\hline & 192,080 & 0,167 & 0,094 & & \\
\hline & 189,660 & 0,196 & 0,094 & & \\
\hline$E$ & 203,150 & 0,166 & 0,086 & \multirow{5}{*}{200,932} & \multirow{5}{*}{1,891} \\
\hline & 198,300 & 0,163 & 0,086 & & \\
\hline & 199,730 & 0,164 & 0,084 & & \\
\hline & 203,020 & 0,168 & 0,086 & & \\
\hline & 200,460 & 0,165 & 0,085 & & \\
\hline \multirow{3}{*}{\multicolumn{3}{|c|}{ Curvatura verticale }} & Raggio curvatura minimo $[\mathrm{cm}]$ & 191,968 & 1,598 \\
\hline & & & Raggio curvatura massimo $[\mathrm{cm}]$ & 200,932 & 1,891 \\
\hline & & & Raggio curvatura medio $[\mathrm{cm}]$ & 195,978 & 2,973 \\
\hline
\end{tabular}

Tab. 2. I raggi d curvatura delle sezioni orizzontali ottenuti attraverso l'algoritmo evoluzionistico.

\begin{tabular}{|c|c|c|c|c|c|}
\hline & & & Sezioni orizzontali & & \\
\hline Sezione & Raggio [cm] & Scarto medio $[\mathrm{cm}]$ & Scarto Quadratico Medio [cm] & Media raggi $[\mathrm{cm}]$ & Scarto Quadratico Medio [cm] \\
\hline \multirow{5}{*}{$\mathrm{F}$} & 580,960 & 0,030 & 0,030 & \multirow{5}{*}{645,306} & \multirow{5}{*}{65,352} \\
\hline & 764,210 & 0,033 & 0,035 & & \\
\hline & 619,130 & 0,031 & 0,028 & & \\
\hline & 662,640 & 0,031 & 0,031 & & \\
\hline & 599,590 & 0,030 & 0,034 & & \\
\hline \multirow[t]{5}{*}{$\bar{G}$} & 603,900 & 0,025 & 0,029 & \multirow{5}{*}{650,668} & \multirow{5}{*}{79,661} \\
\hline & 693,880 & 0,023 & $0,0,020$ & & \\
\hline & 564,290 & 0,027 & 0,036 & & \\
\hline & 605,720 & 0,026 & 0,030 & & \\
\hline & 785,550 & 0,030 & 0,024 & & \\
\hline \multirow[t]{5}{*}{$\mathrm{H}$} & 541,650 & 0,015 & 0,015 & \multirow{5}{*}{625,830} & \multirow{5}{*}{72,371} \\
\hline & 681,190 & 0,021 & 0,022 & & \\
\hline & 646,510 & 0,029 & 0,022 & & \\
\hline & 718,150 & 0,022 & 0,015 & & \\
\hline & 541,650 & 0,015 & 0,015 & & \\
\hline \multirow[t]{5}{*}{$T$} & 729,060 & 0,039 & 0,036 & \multirow{5}{*}{640,416} & \multirow{5}{*}{84,586} \\
\hline & 694,680 & 0,042 & 0,019 & & \\
\hline & 636,150 & $0,0,029$ & $\frac{0,0132}{0,022}$ & & \\
\hline & 658,680 & 0,034 & 0,018 & & \\
\hline & 483,510 & 0,014 & 0,012 & & \\
\hline \multirow[t]{5}{*}{$\bar{L}$} & 817,040 & 0,041 & 0,024 & \multirow{5}{*}{763,500} & \multirow{5}{*}{52,438} \\
\hline & 747,250 & 0,034 & 0,021 & & \\
\hline & 684,600 & 0,030 & 0,019 & & \\
\hline & 742,310 & 0,037 & 0,024 & & \\
\hline & 826,300 & 0,039 & 0,021 & & \\
\hline \multirow[t]{5}{*}{$\mathrm{M}$} & $\frac{020,030}{755,250}$ & $\frac{0,0391}{0,031}$ & $\frac{0,021}{0,030}$ & \multirow{5}{*}{700,816} & \multirow{5}{*}{76,299} \\
\hline & 586,150 & 0,024 & 0,031 & & \\
\hline & 790,360 & 0,033 & 0,034 & & \\
\hline & 733,850 & 0,030 & 0,034 & & \\
\hline & 638,470 & 0,026 & 0,029 & & \\
\hline $\mathrm{N}$ & 627,750 & 0,024 & 0,018 & \multirow{5}{*}{672,414} & \\
\hline & 743,480 & 0,025 & 0,019 & & \\
\hline & 749,740 & 0,027 & 0,018 & & 66,134 \\
\hline & 662,170 & 0,030 & 0,016 & & \\
\hline & 578,930 & 0,025 & 0,017 & & \\
\hline 0 & 722,100 & 0,035 & 0,025 & & \\
\hline & 776,940 & $0,0,039$ & 0 & & \\
\hline & 783,890 & 0,040 & 0,028 & 750,706 & 25,271 \\
\hline & 727,860 & 0,036 & 0,025 & & \\
\hline & 742,740 & 0,040 & 0,029 & & \\
\hline $\bar{P}$ & 682,610 & 0,007 & 0,007 & & \\
\hline & 704,210 & 0,010 & 0,008 & & \\
\hline & 766,510 & 0,012 & 0,011 & 721,264 & 30,051 \\
\hline & 744,220 & 0,010 & 0,010 & & \\
\hline & 708,770 & 0,009 & 00,009 & & \\
\hline $\bar{Q}$ & 518,120 & 0,031 & 0,038 & & \\
\hline & 634,190 & 0,018 & 0,028 & & \\
\hline & 723,760 & 0,014 & 0,018 & 603,248 & 77,006 \\
\hline & 521,490 & 0,031 & 0,024 & & \\
\hline & 618,680 & 0,019 & 0,030 & & \\
\hline Curvatura & rizzontale & & Raggio curvatura minimo $[\mathrm{cm}]$ & 603,248 & 77,006 \\
\hline & & & Raggio curvatura massimo [cm] & 763,500 & 52,438 \\
\hline & & & Raggio curvatura medio $[\mathrm{cm}]$ & 677,417 & 51.531 \\
\hline
\end{tabular}




\section{Riferimenti bibliografici}

Agostini N. (1983). I luoghi di Raffaello a Roma. Roma: Multigrafica Editrice.

Carloni L. (2008). Vincenzo Pagani. Un pittore devoto tra Crivelli e Raffaello. In V. Sgarbi (a cura di). Vincenzo Pagani. Un pittore devoto tra Crivelli e Raffaello. Catalogo della mostra (Fermo, Palazzo dei Priori, 3 I maggio-9 novembre 2008). Cinisello Balsamo: Silvana editoriale, pp. 72-74, cat. 28

Carpo M. (2017). The second digital turn. Design beyond intelligence. Cambridge, London: MIT Press.

Cellini P. (1960). Nota tecnica sul restauro. In Bollettino d'arte, 45, pp. 92-96.

Cheng R., He C., Jin Y., Yao X. (2018). Model-based evolutionary algorithms: a short survey. In Complex \& Intelligent Systems, $n$ 4, pp. 283-292. <https://doi.org/I0. I007/s40747-0 I 8-0080- I> (consultato il 20 febbraio 202 I).

Eslami D., Di Angelo L., Di Stefano P., Pane C. (2020). Review of computer-based methods for archaeological ceramic sherds reconstruction. In Virtual Archaeology Review, n. I I (23), pp. 34-49. <https://doi.org/ I0.4995/var.2020. I 3 I $34>$ (consultato il 20 febbraio 2021).

Giacomini F. (20|4). L'incanto dell'Affresco: capolavori strappati da Pompei a Giotto da Correggio a Tiepolo. In L. Ciancabilla C. Spadoni (a cura di). L'incanto dell'Affresco: capolavori strappati. Catalogo della mostra (Ravenna, Loggetta Lombardesca, 16 febbraio- I 5 giugno 20 I4), vol. I. Cinisello Balsamo. Silvana editoriale, pp. 78-79, cat. 3.

Ginzburg S. (2020). L'Isaia di Raffaello nel I5I3. In Arte Cristiana, vol. CVIII, fasc. 9I7, pp. 90-99.

Mitchell W. J. (1990). The logic of architecture. Design, computation and cognition. Cambridge, London: MIT Press.

Rutten D. (4 marzo 20ll). Evolutionary principles applied to problem solving. <https://ieatbugsforbreakfast.wordpress. com/20 I I/03/04/epatps0 I/> (consultato l'I I febbraio 202 I).

Salerno L. (1960). II Profeta Isaia di Raffaello e il Putto della Accademia di S. Luca. In Bollettino d'arte, 45, pp. 8I-92.

Slowik A., Kwasnicka H. (2020). Evolutionary algorithms and their applications to engineering problems. Neural Computing \& Application, n. 32, pp. 12363-12379. <https://doi.org/l 0.1007/s0052 I-020-04832-8> (consultato il 20 febbraio 202 I).

Ventra S. (2017). «Le plus beau dessin et la plus belle couleur réunis»: il fascino del Putto reggifestone di Raffaello nell'Ottocento. In Accademia Nazionale di San Luca, Annali delle Arti e degli Archivi. Pittura, Scultura, Architettura, 3, pp. 194-200.

Autori

Leonardo Baglioni, Sapienza Università di Roma, leonardo.baglioni@uniromal.it

Marco Fasolo, Sapienza Università di Roma, marco.fasolo@uniromal.it

Matteo Flavio Mancini, matteoflavio.mancini@uniroma3.it

Sofia Menconero, Sapienza Università di Roma, sofia.menconero@uniromal.it

Per citare questo capitolo: Baglioni Leonardo, Fasolo Marco, Mancini Matteo Flavio, Menconero Sofia (202 I). I sistemi evoluzionistici nella ricerca della forma ideale/Evolutionary algorithms in the search for the ideal form. In Arena A., Arena M., Mediati M., Raffa P. (a cura di). Connettere. Un disegno per annodare e tessere Linguaggi Distanze Tecnologie. Atti del $42^{\circ}$ Convegno Internazionale dei Docenti delle Discipline della Rappresentazione/ Connecting. Drawing for weaving relationship. Languages Distances Technologies. Proceedings of the $42^{\text {th }}$ International Conference of Representation Disciplines Teachers. Milano: FrancoAngeli, pp. $159-178$ 


\title{
Evolutionary Algorithms in the Search for the Ideal Form
}

\author{
Leonardo Baglioni \\ Marco Fasolo \\ Matteo Flavio Mancini \\ Sofia Menconero
}

Abstract

The interpretative hypotheses on the original form can be formulated and verified using ideal geometric models capable of continuously representing the study's morphogenetic characteristics. From a procedural point of view, this phase corresponds to the translation of an acquired data, described by a discrete language, to an interpretative one, represented through a continuous language.

The present study aims to propose an investigation on the potential of evolutionary computational methods (Evolutionary Solver or Genetic Algorithm) applied to the interpretation phase and the ideal form's finding.

These algorithms, used to understand the morphogenetic characteristics of a fresco fragment attributed to Raphael, have allowed not only to hypothesise the measure of the radius of the longitudinal curvature, which is already visible at a direct observation but also to highlight a second curvature, an horizontal and hardly perceptible one.

Keywords evolutionary solver, descriptive geometry, Image-Based Survey Methods, fresco, Raffaello.

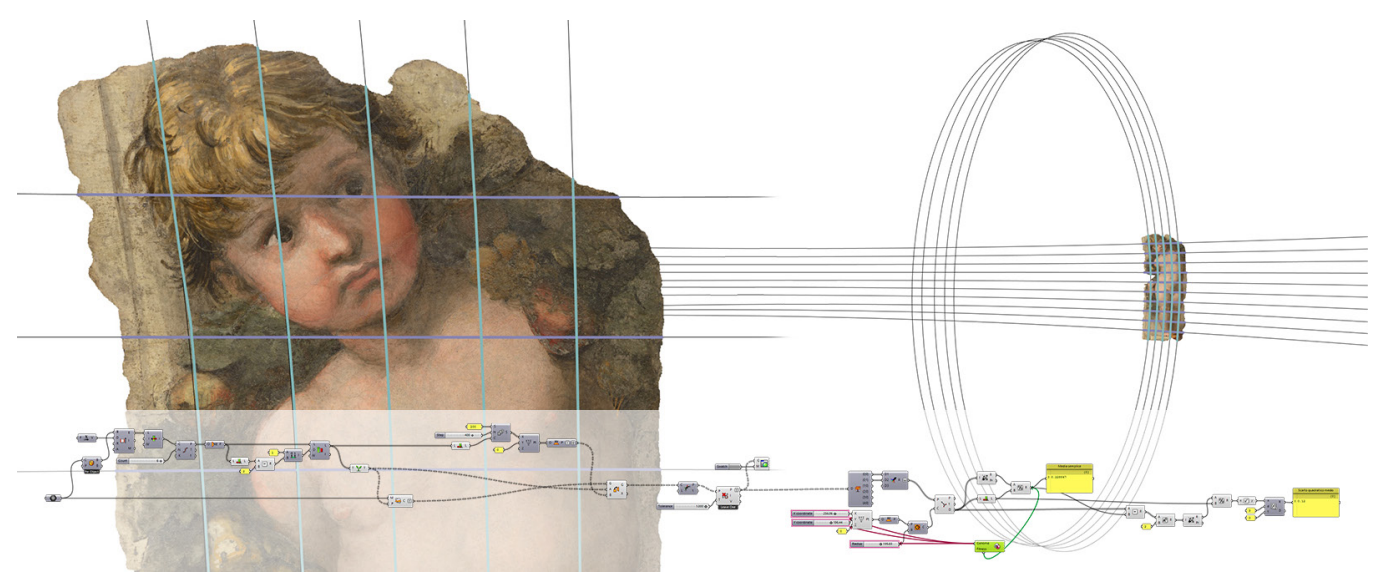




\section{Introduction}

Surveying, intended as a knowledge operation aimed at the deep understanding of an artefact, defines a path articulated according to different phases and procedures coordinated and integrated depending on the nature of the object and the study's purposes. The entire process is oriented towards defining a cognitive model capable of representing the evolutions and metamorphoses that have affected the asset over time, starting from the project's initial idea up to the current state of conservation. Thanks to technological advances in recent years, the measurement phase has become a massive acquisition operation aimed above all at documenting the object's surface. The consequences of this approach are that, in some cases, surveying has become a technical and technological activity rather than a critical and interpretative one.

The original form's interpretative hypotheses can only be formulated and verified through ideal geometric models capable of continuously representing the object's morphogenetic characteristics. This interpretative operation is not an easy task due to several aspects: on the one hand, the nature of the object and the vicissitudes that involved it, and on the other, the interdisciplinary and interpretative nature of the operation. From a procedural point of view, this phase corresponds to the transformation of data, acquired and described with a discrete language, into an interpretative one represented through a continuous language. The present study is part of this context, which is particularly important for restoring the dignity of surveying as the knowledge activity to which we have referred by proposing an investigation of the potential of evolutionary computational methods aimed at finding the ideal form.

These methods were introduced in the early 1960s, but only recently they have become widespread thanks to the development of open platforms that allow them to be used by non-programmers. Evolutionary Solver or Genetic Algorithm systems in which evolutionary logics are addressed to the solution of specific problems are characterised by the extreme flexibility with which they can be applied in a wide variety of problems [Cheng 20 I 8, Slowik 2020]. The calculation process is based on modelling in which the variables are represented by the genes that characterise the individuals (called genomes) that populate the fitness landscape (the multidimensional model in which the calculation will take place). By varying the genes, the state of the model, and consequently, the final fitness will change, for better or worse, depending on the calculation's objectives (fig. I).

Fig. I.Typical scheme of an evolutionary algorithm: populating the landscape with a random set of individuals; evaluating each individual and creating individual and creating a hierarchical order; creating a new generation of individuals from the selection made on the
initial generation.

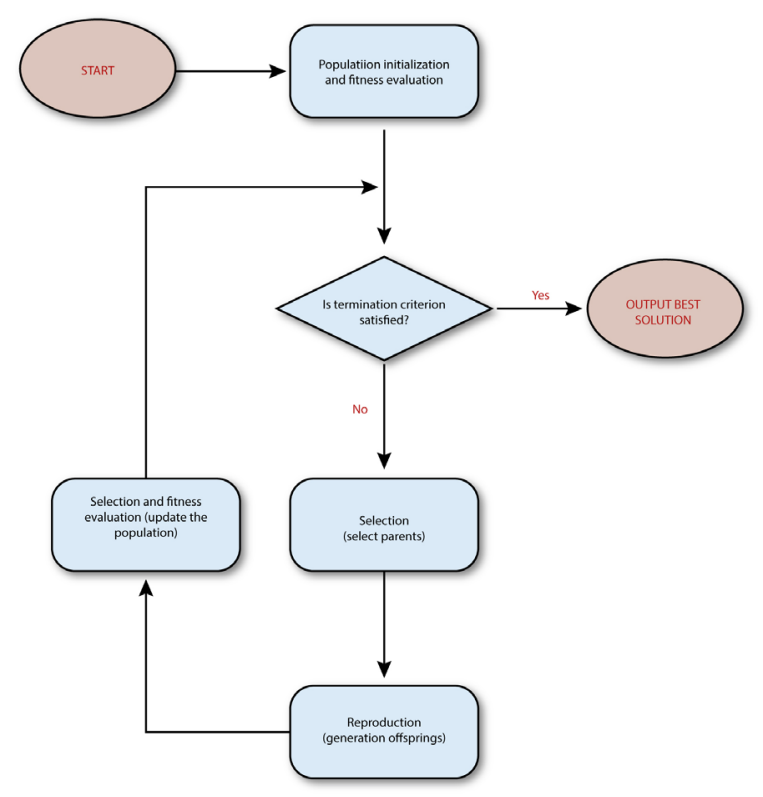


However, what is the 'best result'? The answer to this apparently trivial question defines a fundamental characteristic of Evolutionary Algorithms: these computational methods do not guarantee a single solution. Until a reference value is specified on what can be considered 'good enough', the process would be repeated indefinitely without ever reaching an exact answer.

Despite this limit that can be overcome only with a series of user-defined choices that give a critical character to the whole operation, Evolutionary Algorithms can offer remarkable contributions in the interpretation phase of a surveyed form.

A case study to test this methodology has been offered to us by collaborating with the Accademia Nazionale di San Luca, which has put an asset of its property at our disposal to carry out some geometric investigations on it. The object in question is the Putto reggifestone attributed to Raphael, which was donated to San Luca in 1834 through a bequest by Jean Baptiste Wicar, a leading figure of the Academy [I].

It is a fragment of painting on a plaster of about $40 \times 107 \mathrm{~cm}$ attached to masonite support connected to a tablet through iron brackets. The fragment's surface appears to be curved in the longitudinal direction, while a second curvature, not visible to the naked eye, affects the horizontal section, as we will demonstrate.

\section{From real to ideal form}

The fresco fragment's acquisition was planned to prioritise the geometric and chromatic control, following considerations on the object's nature and the study's purposes aimed at morphological knowledge. From this point of view, and since the object's dimensions were not particularly large, the photogrammetric technique allowed both objectives to be achieved in the manner described below.

It was essential to have accurate geometric survey data, both in terms of general formal correspondence and micromorphology resolution, to carry out morphogenetic studies.

The photographic acquisition included several sessions to achieve this double objective: a series of overall shots with a $50 \mathrm{~mm}$ lens to capture the entire object and the targets,
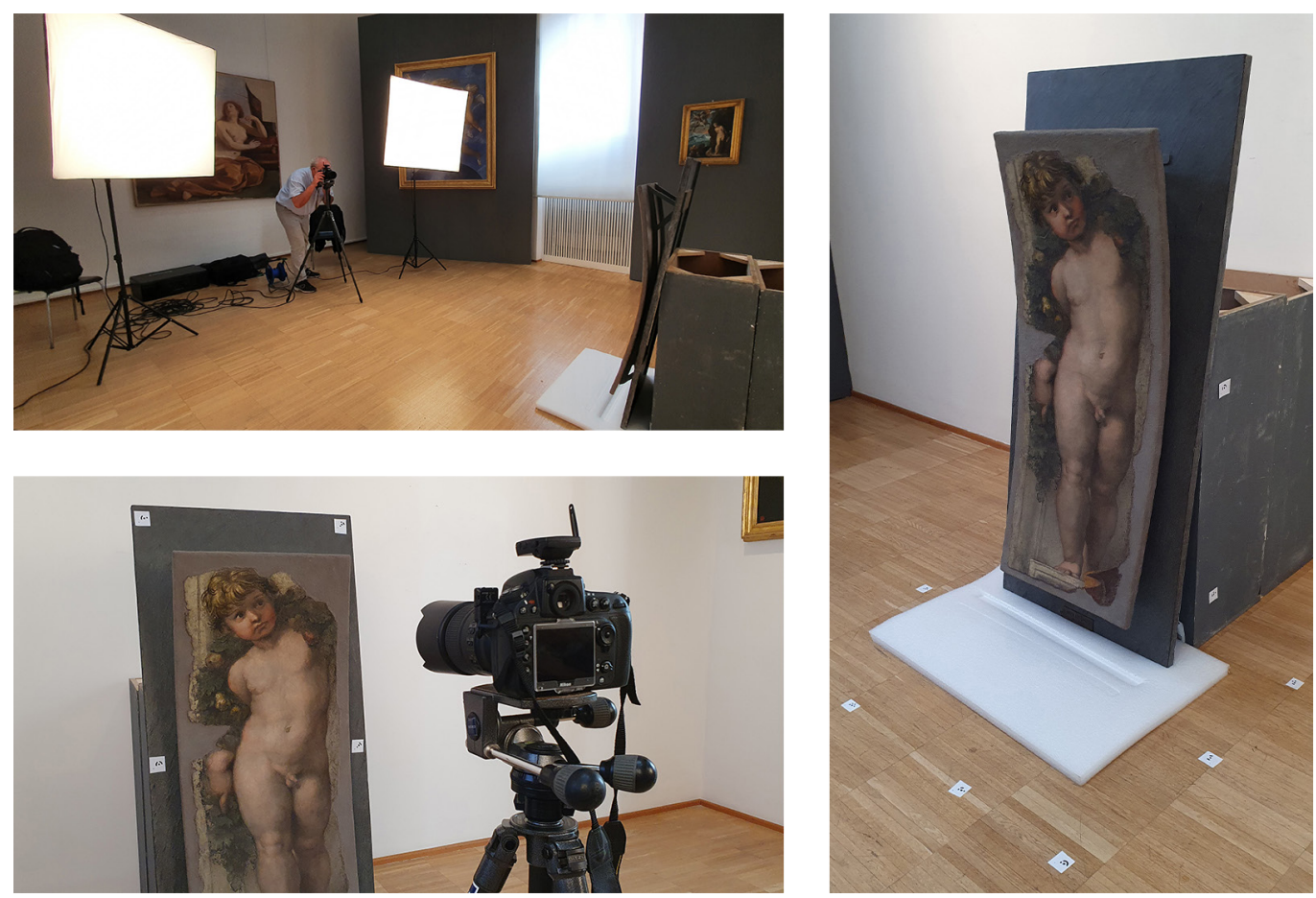
intending to ensure a solid geometric structure for the alignment of the subsequent photographs; other closer shots with the same lens aimed at the detailed description of the micromorphology; a series of shots with a $105 \mathrm{~mm}$ lens to capture the figurative detail at high resolution (fig. 2).

The photographic data processing was supported metrically by the insertion of I 8 control points (targets) positioned in a distributed manner on the surfaces adjacent to the fresco fragment and whose distances were measured directly. Of the 20 measurements taken, 12 were used to scale the model and the remaining 8 as control measurements (fig. 3). The point cloud, cleaned and trimmed to isolate the fresco fragment's limits, counts 3,324,005 points, and a surface of 2,000,000 polygons was obtained from it (fig. 4).

A minimal reprojection error on the individual targets (average deviation of $0.17 \mathrm{~mm}$ ), and a high level of detail of the micromorphology of the mesh surface, declared by the recording of the boundaries between two giornate (day's work) (fig. 5), guaranteed the geometric control of the results.
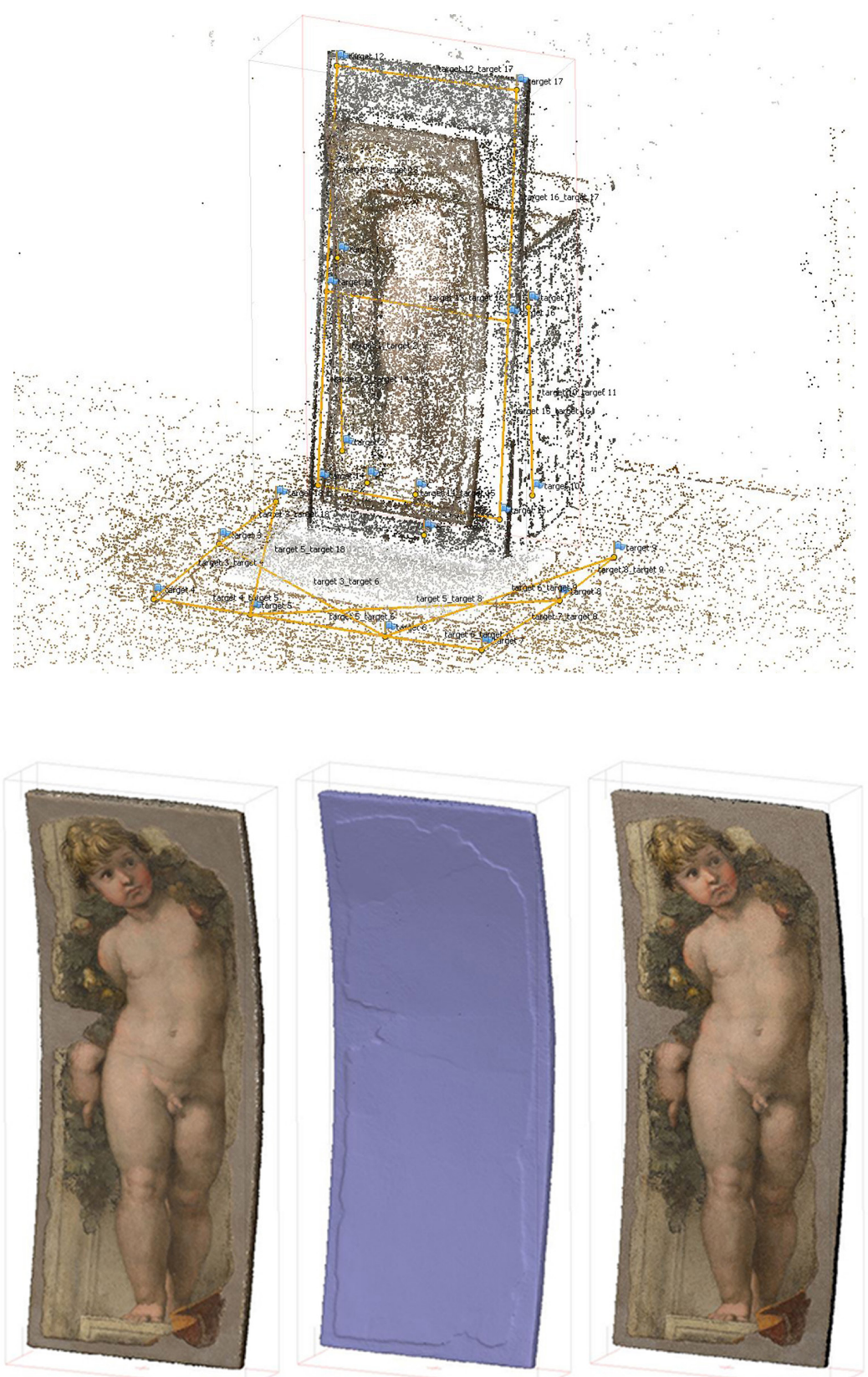
Fig. 5. Detail of the lower part of the mesh model showing the boundaries (day's work).
Because of the object's artistic nature, the geometric control was accompanied by an equally high-quality colour rendering.

The diffuse and controlled lighting set up, together with the white balance procedure and detail shots with a $105 \mathrm{~mm}$ lens (best for resolution and sharpness), resulted in a texture of $8,192 \times 8,192$ pixels that was so well defined that it was possible to observe the evidence of the cartoon transfer process (fig. 6).

The data obtained through the photogrammetric survey is a mesh, a surface defined by a discrete polyhedral structure. The study of the geometric characteristics was conducted through the analysis of vertical and horizontal sample sections generated through the inter-
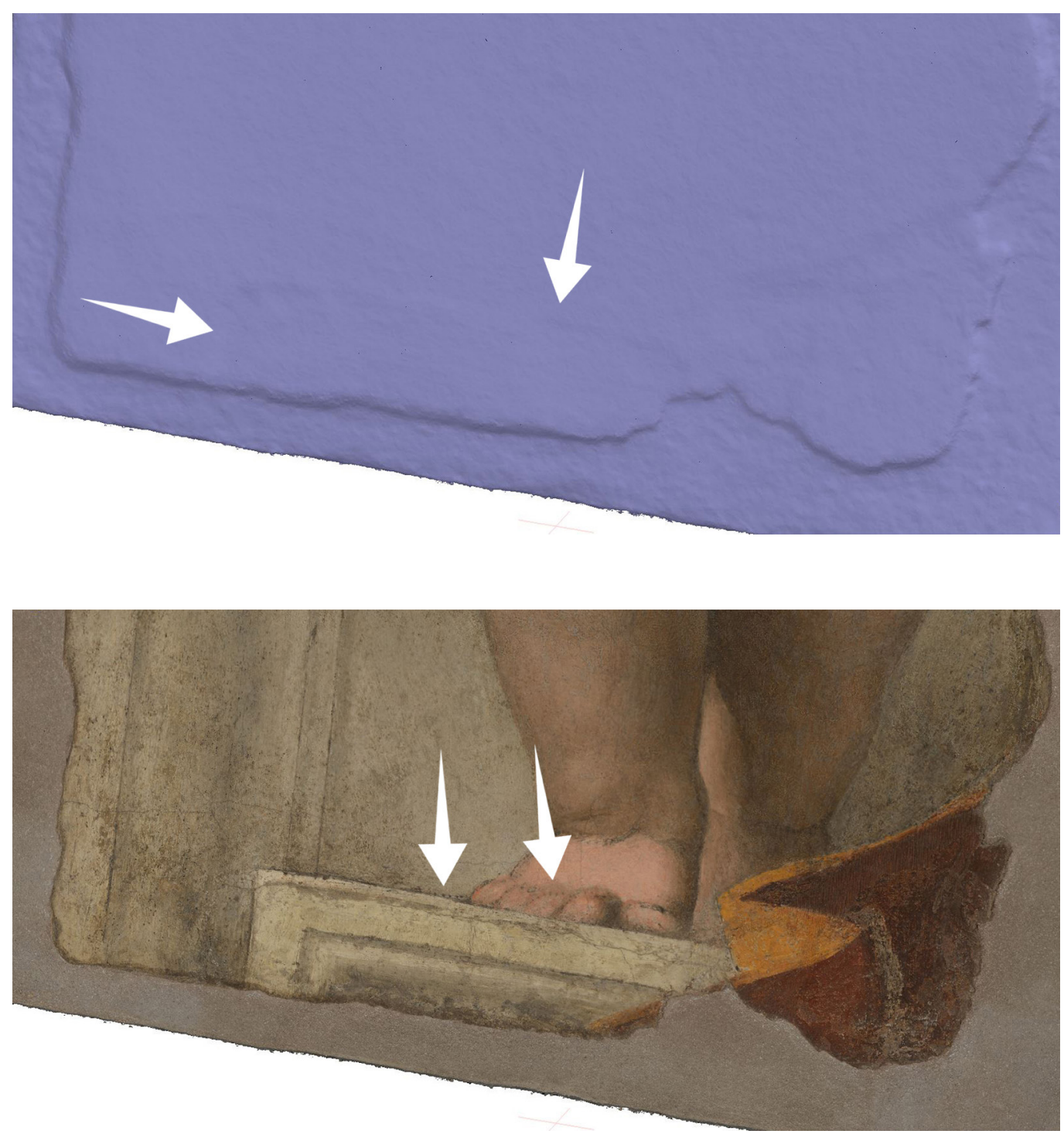

section with two parallel plane stacks, which provided: five vertical sections (A-E) and ten horizontal sections (F-Q) (fig. 7). Each section is represented by a polyline whose vertices were sampled at a distance of $\mathrm{I} \mathrm{cm}$.

It is necessary to choose a geometric reference model, which in this case was the circumference, to determine the curvature radius of a discrete set of points. In fact, circumference is one of the most common generating curves in historical architecture, and it univocally gives 
Fig. 7. The five vertical sections (A-E) and ten horizontal sections ( $\mathrm{F}-\mathrm{Q}$ ) and their respective circumferences calculated.
Fig. 8. The functional blocks of the algorithm: (a) generation of the sections; (b) rotating of the sections on the $x y$ construction plane; (c) sampling of the vertices of the sections: (d) finding the bestions; (d) finding chest approximation circumferences of the sections, (e) calculation of the mean square deviation.
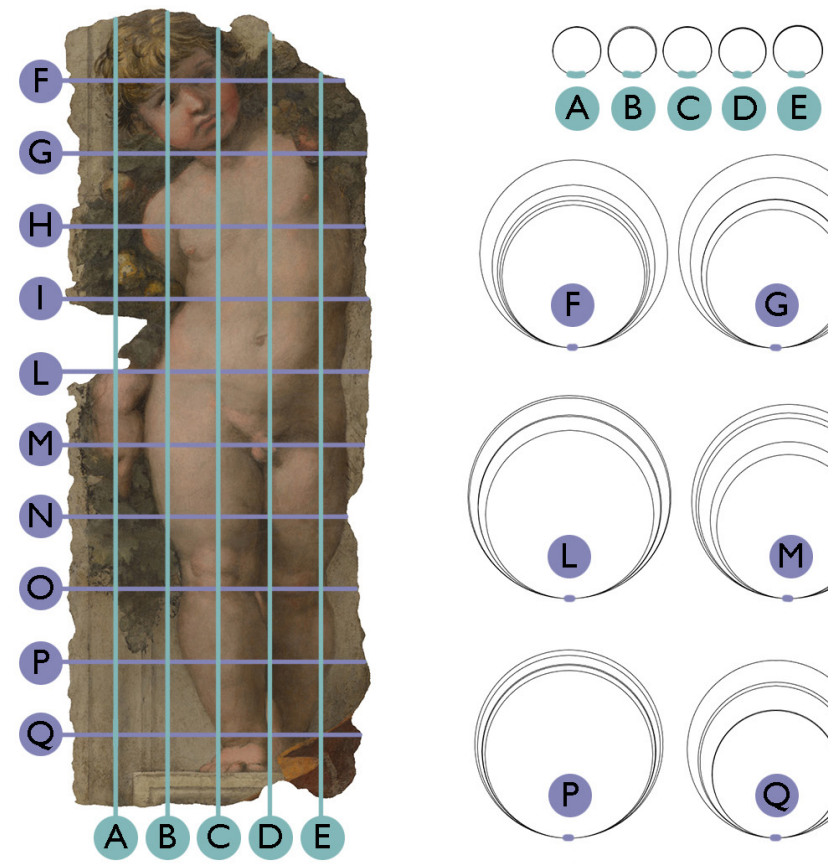

A B C D E
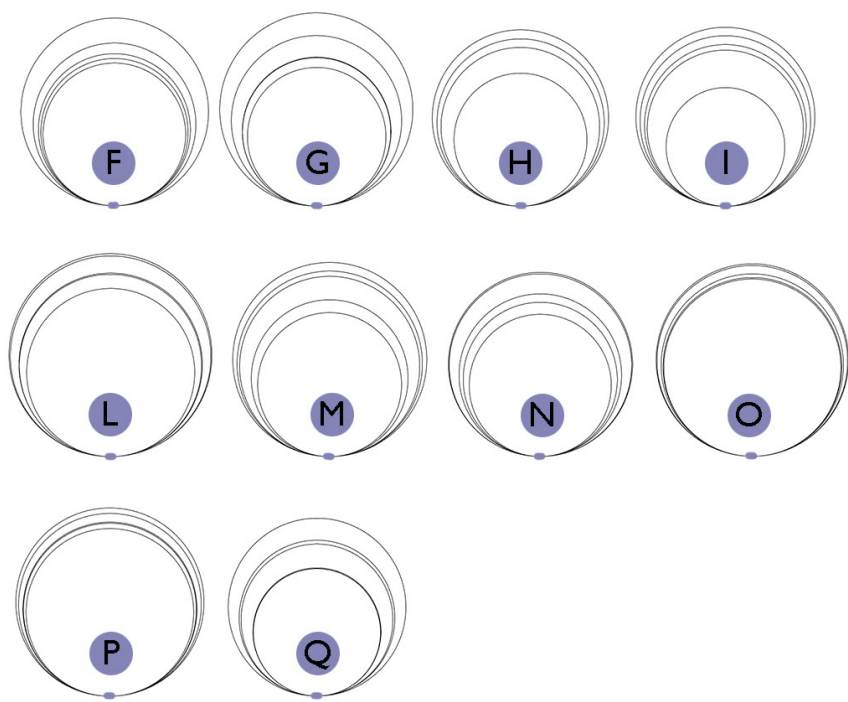

back the sought property through the value of its radius, once defined as the best approximation circumference of the given points.

In this experimentation, an algorithmic procedure was used, programmed using Visual Programming Language (VPL) tools to manage all the processing [2] (fig. 8). The definition of the best approximation circumferences adopted a 'generate-and-test' approach, i. e. an evolutionary criterion. The solutions' generation takes place in a semi-random way to be subsequently subjected to a rigorous critical selection [Mitchell 1990, pp. 179, I80].

This heuristic procedure is increasingly applied, exploiting the computational potential available today to digitally simulate a population of prototypes among which looking for the optimal solution or, rather, the best available in a given number of iterations, according to the "search don't sort" paradigm that increasingly pervades the second digital turn of Big Data [Carpo 2017, pp. 23-55].

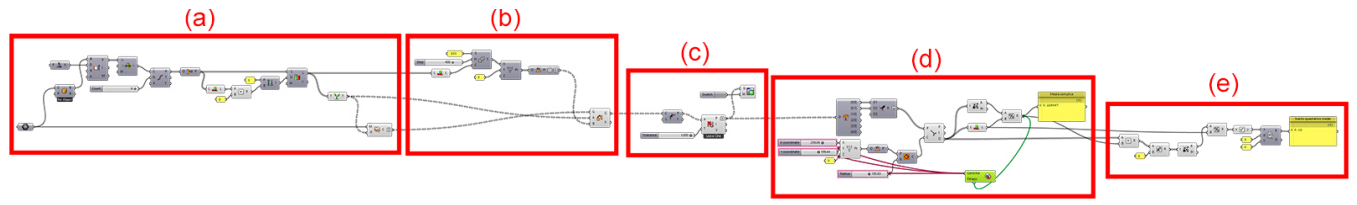

A genome $(x, y, R)$, defined by the Cartesian coordinates of the centre and the radius's value, characterise each circumference. The evolutionary algorithm searches for the triad corresponding to the best approximation circumference of the analysed points, i.e. the circumference that minimises the sum of the distances from the polyline section's vertices. Some critical choices are necessary to set and orient the evolutionary process: to decide the number of individuals of each generation ( 100 ); to indicate the percentage of individuals that are candidates to generate the next generation (I0\%); to establish the percentage of endogamy, i.e. whether to allow the coupling of individuals with similar genomes or to favour the coupling between different genomes (-75\%); to establish after how many occurrences of the same genome this is chosen as the most satisfactory and to end the calculation ( 100$)$. 
The percentage of endogamy in the pairings is significant: if this condition is allowed, convergence towards a result will be faster, but the risk of obtaining a false positive in the case of problems with more than one possible solution will increase; on the contrary, if endogamy is prevented, convergence will be slower, but the more significant variability of the genome will result in a lower probability of false positives (fig. 9).

The results provided by the evolutionary algorithms are not univocal. They can show oscillations between successive repetitions, so each section was tested five times and, for each reiteration, the mean value of the distances between the circumference and the vertices of the polyline and their mean square error (standard deviation) were calculated. The latter allows the distribution of the distances around the mean value to be assessed and thus the

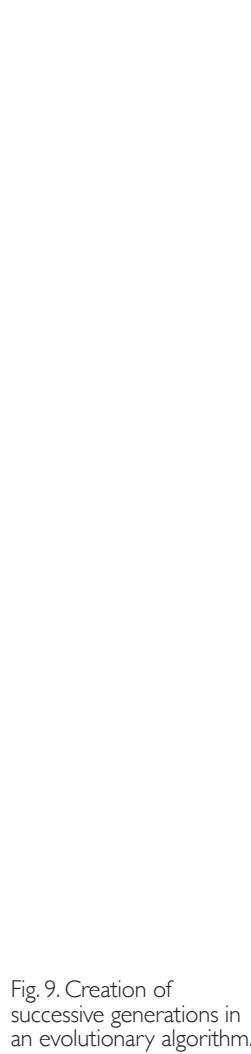

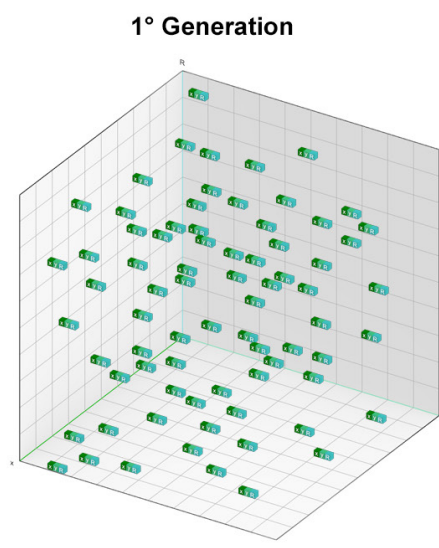

Classifing genomas based on their fitness

1. $(x, y, R)$

2. $(x, y, R)_{2}$

3. $(x, y, R)_{3}$

4. $(x, y, R)_{4}$

5. $(x, y, R)_{5}$

6. $(x, y, R)_{6}$

7. $(x, y, R)_{7}$

8. $(x, y, R)_{8}$

9. $(x, y, R)_{9}$

10. $(x, y, R)$

$\cdots$

n. $(x, y, R)_{n}$ $2^{\circ}$ Generation

High imbreeding
$(0 \%->+100 \%)$

$(x, y, R)_{1}+(x, y, R)_{2}$

$(x, y, R)_{1}+(x, y, R)_{3}$

$(x, y, R)_{3}+(x, y, R)_{4}$

...

Imbreeding

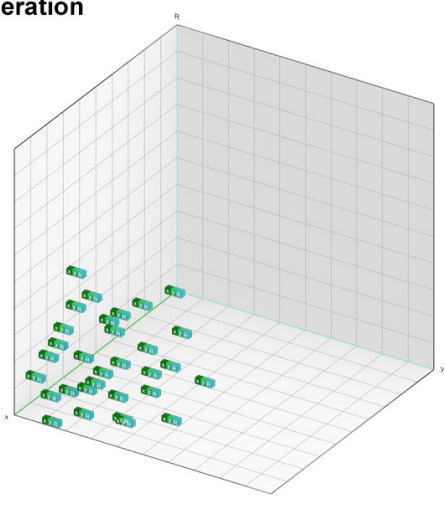

Low imbreeding

(0\% -> - 100\%)

$(x, y, R)_{1}+(x, y, R)_{10}$

$(x, y, R)_{1}+(x, y, R)_{8}$

$(x, y, R)_{2}+(x, y, R)_{9}$

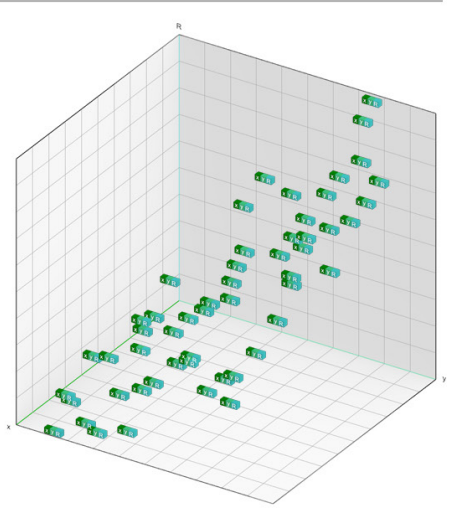

result's consistency (figs. I0, I I). For each section, the mean value of the five identified circumferences' radii and their mean square deviation were then calculated.

The vertical sections (A-E) provided precise and accurate results, giving a reliable indication of the average vertical curvature radius, which was $195.978 \mathrm{~cm}$, with a mean square deviation of $2.973 \mathrm{~cm}$, equal to $1.52 \%$ of the average radius (Table I).

The generation of the horizontal sections ( $\mathrm{F}-\mathrm{Q}$ ) revealed a second curvature, which is difficult to perceive by direct observation, and was, therefore, subject to the same analysis carried out for the vertical sections.

In the latter case, the results show considerable fluctuations even in the single readings, and this harms the average horizontal curvature radius, which is $677.417 \mathrm{~cm}$, but with a mean square deviation of $51.531 \mathrm{~cm}$, equal to $7.61 \%$ of the average radius, a significantly high value (Table 2). This result can be ascribed to the analysed data nature, which is characterised both by a slight curvature value and a reduced extension of the fragment horizontal dimension. The results we have described were verified by analysing the same data with statistical analysis algorithms based on best fitting computation, using the circumference equation as 
a model. The results are, in fact, coincident as the differences between the deviations are negligible. Therefore, the results obtained through VPL were preferred because of the better optimisation of the returned deviations and because this technique allows better control of the whole process.

Fig. 10. Vertical section $A$ and a detail of the approximation (mean radius $196.346 \mathrm{~cm}$; mea square deviation 0.737 $\mathrm{cm})$.

Fig. I I. Horizontal section $L$ and a detail of the approximation (mean radius $736.500 \mathrm{~cm}$; mean square deviation 52 . $438 \mathrm{~cm})$.
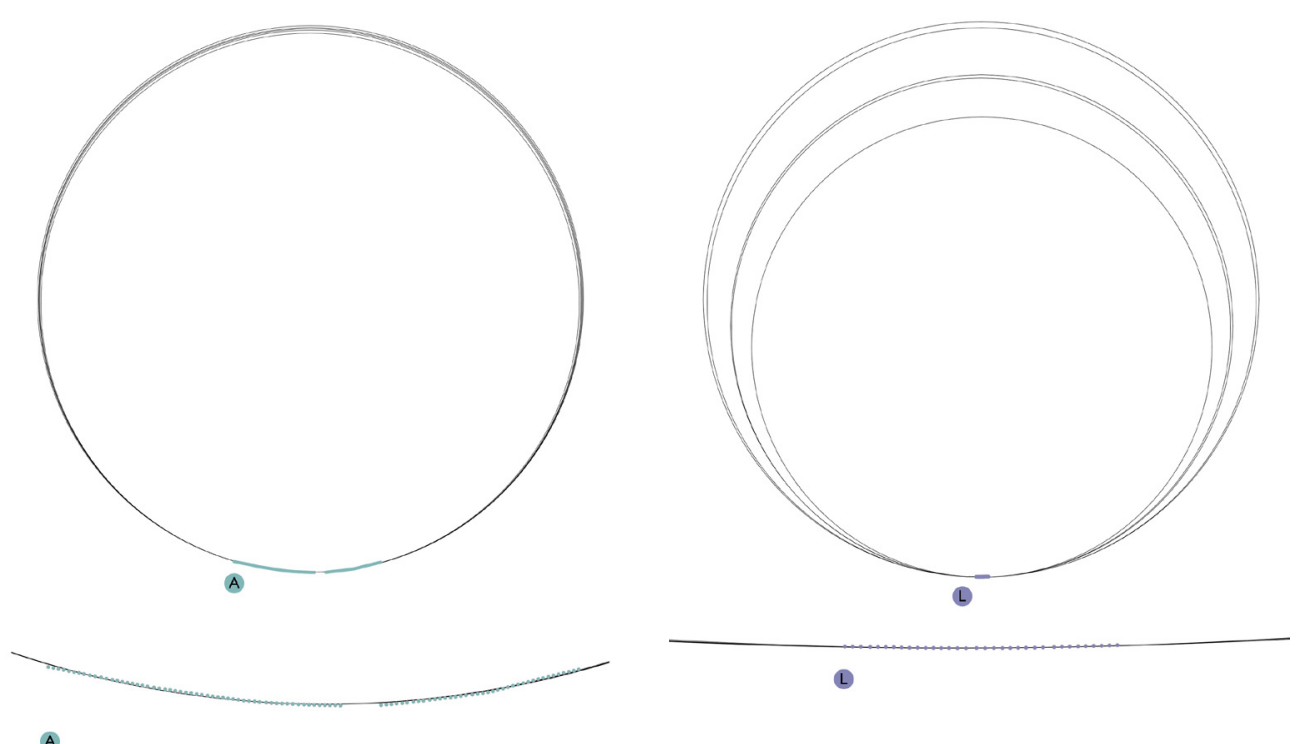

\section{Conclusions}

The study demonstrated the potential of evolutionary algorithms as a tool for geometric analysis to interpret the form. The analyses carried out on the fresco fragment have highlighted a second horizontal curvature that accompanies the first, more evident vertical one. The result achieved leads us not to exclude the possibility that the fragment can be considered a portion of the surface with double curvature or, rather, with positive Gaussian curvature.

The application of these algorithms to translation from a discrete to a continuous modelling language should not be considered purely automated. Both the choice of the geometric model of reference and the alternatives in setting the rules governing the creation of the generations required specialised scientific knowledge and the ability to make critical choices typical of the interpretative phases. The decision of the geometric model of reference is a typical operation of differential geometry, which studies a form in relation to other simpler forms with known properties.

The potential that emerged in the experimentation opens up its possible developments toward the use of two-dimensional geometric primitives and its application in sectors such as archaeological and architectural reconstructions [Eslami et al. 2020], which are more often implemented through other analogue and digital techniques.

\section{Notes}

[I] For the history of the Putto reggifestone, such as its authenticity, attribution and original provenance, please refer to the following studies [Agostini 1983; Carloni 2008; Cellini 1960; Giacomini 20 I4; Ginzburg 2020; Salerno 1960; Ventra 20I7].

[2] The visual programming language Grasshopper, implemented in the McNeel Rhinoceros 6.0 three-dimensional modelling software, and its evolutionary calculation engine Galapagos were used. 


\section{Acknowledgements}

We want to thank the Accademia Nazionale di San Luca for allowing the Putto reggifestone surveys and Prof. Riccardo Migliari for advising and supervising the study.

This contribution resulted from a collaboration between the authors, who agreed to assign themselves the following activities: Leonardo Baglioni and Marco Fasolo supervised the work, while Sofia Menconero was responsible for the photogrammetric survey and Matteo Flavio Mancini for the geometric analysis of the fragment.

Tab. I. The radii of curvature of vertica sections obtained

through the evolutionary algorithm.
Tab. 2. The radii of curvature of horizontal sections obtained

through the evolutionary algorithm.

\begin{tabular}{|c|c|c|c|c|c|}
\hline \multicolumn{6}{|c|}{ Sezioni verticali } \\
\hline Sezione & Raggio $[\mathrm{cm}]$ & Scarto medio $[\mathrm{cm}]$ & Scarto Quadratico Medio [cm] & Media raggi $[\mathrm{cm}]$ & Scarto Quadratico Medio $[\mathrm{cm}]$ \\
\hline \multirow[t]{5}{*}{$A$} & 195,830 & 0,228 & 0,120 & \multirow{5}{*}{196,346} & \multirow{5}{*}{ 年 } \\
\hline & 196,740 & 0,225 & 0,124 & & \\
\hline & 197,470 & 0,232 & 0,117 & & \\
\hline & 196,360 & 0,227 & 0,117 & & \\
\hline & 195,330 & 0,231 & 0,128 & & \\
\hline \multirow[t]{5}{*}{ B } & 194,100 & 0,227 & 0,118 & \multirow{5}{*}{194,190} & \multirow{5}{*}{1,329} \\
\hline & 193,750 & 0,230 & 0,118 & & \\
\hline & 196,690 & 0,241 & 0,127 & & \\
\hline & 193,670 & 0,228 & 0,120 & & \\
\hline & 192,740 & 0,226 & 0,119 & & \\
\hline \multirow[t]{5}{*}{$\mathrm{c}$} & 197,080 & 0,204 & 0,115 & \multirow{5}{*}{196,456} & \multirow{5}{*}{1,157} \\
\hline & 196,490 & 0,208 & 0,113 & & \\
\hline & 195,500 & 0,201 & 0,113 & & \\
\hline & 194,970 & 0,203 & 0,113 & & \\
\hline & 198,240 & 0,212 & 0,114 & & \\
\hline \multirow[t]{5}{*}{$\bar{D}$} & 190,750 & 0,154 & 0,098 & \multirow{5}{*}{191,968} & \multirow{5}{*}{1,598} \\
\hline & 193,920 & 0,170 & 0,095 & & \\
\hline & 193,430 & 0,165 & 0,095 & & \\
\hline & 192,080 & 0,167 & 0,094 & & \\
\hline & 189,660 & 0,196 & 0,094 & & \\
\hline$E$ & 203,150 & 0,166 & 0,086 & \multirow{5}{*}{200,932} & \multirow{5}{*}{1,891} \\
\hline & 198,300 & 0,163 & 0,086 & & \\
\hline & 199,730 & 0,164 & 0.084 & & \\
\hline & 203,020 & 0,168 & 0,086 & & \\
\hline & 200,460 & 0,165 & 0,085 & & \\
\hline \multirow{3}{*}{\multicolumn{3}{|c|}{ Curvatura verticale }} & Raggio curvatura minimo $[\mathrm{cm}]$ & 191,968 & 1,598 \\
\hline & & & Raggio curvatura massimo $[\mathrm{cm}]$ & 200,932 & 1,891 \\
\hline & & & Raggio curvatura medio [cm] & 195,978 & 2,973 \\
\hline
\end{tabular}

\begin{tabular}{|c|c|c|c|c|c|}
\hline \multicolumn{6}{|c|}{ Sezioni orizzontali } \\
\hline Sezione & Raggio $[\mathrm{cm}]$ & Scarto medio [cm] & Scarto Quadratico Medio [cm] & Media raggi $[\mathrm{cm}]$ & Scarto Quadratico Medio $[\mathrm{cm}]$ \\
\hline \multirow[t]{5}{*}{$\bar{F}$} & 580,960 & 0,030 & 0,030 & \multirow{5}{*}{645,306} & \multirow{5}{*}{65,352} \\
\hline & 764,210 & 0,033 & 0,035 & & \\
\hline & 619,130 & 0,031 & 0,028 & & \\
\hline & 662,640 & 0,031 & 0,031 & & \\
\hline & 599,590 & 0,030 & 0,034 & & \\
\hline \multirow[t]{5}{*}{$\bar{G}$} & 603,900 & 0,025 & 0,029 & \multirow{5}{*}{650,668} & \multirow{5}{*}{79,661} \\
\hline & 693,880 & 0,023 & 0,020 & & \\
\hline & 564,290 & 0,027 & 0,036 & & \\
\hline & 605,720 & 0,026 & 0,030 & & \\
\hline & 785,550 & 0.030 & 0.024 & & \\
\hline \multirow[t]{5}{*}{$\mathrm{H}$} & 541,650 & 0,015 & 0,015 & \multirow{5}{*}{625,830} & \multirow{5}{*}{72,371} \\
\hline & 681,190 & 0,021 & 0,022 & & \\
\hline & 646,510 & 0,029 & 0,022 & & \\
\hline & 718,150 & 0,022 & 0,015 & & \\
\hline & 541,650 & 0,015 & 0,015 & & \\
\hline \multirow[t]{5}{*}{1} & 729,060 & 0,039 & 0,036 & \multirow{5}{*}{640,416} & \multirow{5}{*}{84,586} \\
\hline & 694,680 & 0,042 & 0,019 & & \\
\hline & 636,150 & 0,029 & 0,022 & & \\
\hline & 658,680 & 0,034 & 0,018 & & \\
\hline & 483,510 & 0,014 & 0,012 & & \\
\hline \multirow[t]{5}{*}{$\mathrm{L}$} & 817,040 & 0,041 & 0,024 & \multirow{5}{*}{763,500} & \multirow{5}{*}{52,438} \\
\hline & 747,250 & 0,034 & 0,021 & & \\
\hline & 684,600 & 0,030 & 0,019 & & \\
\hline & 742,310 & 0,037 & 0,024 & & \\
\hline & 826,300 & 0,039 & 0,021 & & \\
\hline \multirow[t]{5}{*}{ M } & 755,250 & 0,031 & 0,030 & \multirow{5}{*}{700,816} & \multirow{5}{*}{76,299} \\
\hline & 586,150 & 0,024 & 0,031 & & \\
\hline & 790,360 & 0,033 & 0,034 & & \\
\hline & 733,850 & 0,030 & 0,034 & & \\
\hline & 638,470 & 0,026 & 0,029 & & \\
\hline $\mathrm{N}$ & 627,750 & 0,024 & 0,018 & & \\
\hline & 743,480 & 0,025 & 0,019 & & \\
\hline & 749,740 & 0,027 & 0,018 & 672,414 & 66,134 \\
\hline & 662,170 & 0,030 & 0,016 & & \\
\hline & 578,930 & 0.025 & 0.017 & & \\
\hline 0 & 722,100 & 0,035 & 0,025 & & \\
\hline & 776,940 & 0,039 & 0,029 & & \\
\hline & 783,890 & 0,040 & 0,028 & 750,706 & 25,271 \\
\hline & 727,860 & 0,036 & 0,025 & & \\
\hline & 742,740 & 0,040 & 0,029 & & \\
\hline$P$ & 682,610 & 0,007 & 0,007 & & \\
\hline & 704,210 & 0,010 & 0,008 & & \\
\hline & 766,510 & 0,012 & 0,011 & 721,264 & 30,051 \\
\hline & 744,220 & 0,010 & 0.010 & & \\
\hline & 708,770 & 0,009 & 0,009 & & \\
\hline $\bar{Q}$ & 518,120 & 0,031 & 0,038 & & \\
\hline & 634,190 & 0,018 & 0,028 & & \\
\hline & 723,760 & 0,014 & 0,018 & 603,248 & 77,006 \\
\hline & 521,490 & 0,031 & 0,024 & & \\
\hline & 618,680 & 0,019 & 0,030 & & \\
\hline curvatura & $\overline{z z o n t a l e}$ & & Raggio curvatura minimo $[\mathrm{cm}]$ & 603,248 & 77,006 \\
\hline & & & Raggio curvatura massimo $[\mathrm{cm}]$ & 763,500 & 52,438 \\
\hline & & & Raggio curvatura medio $[\mathrm{cm}]$ & 677,417 & 51,531 \\
\hline
\end{tabular}




\section{References}

Agostini N. (1983). I luoghi di Raffaello a Roma. Roma: Multigrafica Editrice.

Carloni L. (2008). Vincenzo Pagani. Un pittore devoto tra Crivelli e Raffaello. In V. Sgarbi (a cura di). Vincenzo Pagani. Un pittore devoto tra Crivelli e Raffaello. Catalogo della mostra (Fermo, Palazzo dei Priori, 3 I maggio-9 novembre 2008). Cinisello Balsamo: Silvana editoriale, pp. 72-74, cat. 28

Carpo M. (2017). The second digital turn. Design beyond intelligence. Cambridge, London: MIT Press.

Cellini P. (1960). Nota tecnica sul restauro. In Bollettino d'arte, 45, pp. 92-96.

Cheng R., He C., Jin Y., Yao X. (20 I 8). Model-based evolutionary algorithms: a short survey. In Complex \& Intelligent Systems, n. 4, pp. 283-292. <https://doi.org/10.1007/s40747-0 I 8-0080-I> (accessed on 202 I, Febraury 20)

Eslami D., Di Angelo L., Di Stefano P., Pane C. (2020). Review of computer-based methods for archaeological ceramic sherds reconstruction. In Virtual Archaeology Review, n. I I (23), pp. 34-49. <https://doi.org/I0.4995/var.2020.13134> (accessed on 2021, Febraury 20).

Giacomini F. (20|4). L'incanto dell'Affresco: capolavori strappati da Pompei a Giotto da Correggio a Tiepolo. In L. Ciancabilla, C. Spadoni (a cura di). L'incanto dell'Affresco: capolavori strappati. Catalogo della mostra (Ravenna, Loggetta Lombardesca, I6 febbraio- 15 giugno 20 I 4), vol. I. Cinisello Balsamo. Silvana editoriale, pp. 78-79, cat. 3.

Ginzburg S. (2020). L'Isaia di Raffaello nel I 5 I3. In Arte Cristiana, vol. CVIII, fasc. 917, pp. 90-99.

Mitchell W. J. (1990). The logic of architecture. Design, computation and cognition. Cambridge, London: MIT Press.

Rutten D. (4 marzo 201I). Evolutionary principles applied to problem solving. <https://ieatbugsforbreakfast.wordpress. com/20 I l/03/04/epatps0 I/> (accessed on 202 I, Febraury I I).

Salerno L. ( 1960). II Profeta Isaia di Raffaello e il Putto della Accademia di S. Luca. In Bollettino d'arte, 45, pp. 81-92.

Slowik A., Kwasnicka H. (2020). Evolutionary algorithms and their applications to engineering problems. Neural Computing \& Application, n. 32, pp. I 2363-1 2379. <https://doi.org/1 0.1007/s0052 I-020-04832-8> (accessed on 2021, Febraury 20).

Ventra S. (2017). «Le plus beau dessin et la plus belle couleur réunis»: il fascino del Putto reggifestone di Raffaello nell'Ottocento. In Accademia Nazionale di San Luca, Annali delle Arti e degli Archivi. Pittura, Scultura, Architettura, 3, pp. 194-200.

\section{Authors}

Leonardo Baglioni, Sapienza Università di Roma, leonardo.baglioni@uniromal .it

Marco Fasolo, Sapienza Università di Roma, marco.fasolo@uniromal.it

Matteo Flavio Mancini, matteoflavio.mancini@uniroma3.it

Sofia Menconero, Sapienza Università di Roma, sofia.menconero@uniromal.it

To cite this chapter. Baglioni Leonardo, Fasolo Marco, Mancini Matteo Flavio, Menconero Sofia (202I). I sistemi evoluzionistici nella ricerca della forma ideale/Evolutionary algorithms in the search for the ideal form. In Arena A., Arena M., Mediati D., Raffa P. (a cura di). Connettere. Un disegno per annodare e tessere Linguaggi Distanze Tecnologie. Atti del $42^{\circ}$ Convegno Internazionale dei Docenti delle Discipline della Rappresentazione/ Connecting. Drawing for weaving relationship. Languages Distances Technologies. Proceedings of the $42^{\text {th }}$ International Conference of Representation Disciplines Teachers. Milano: FrancoAngeli, pp. $159-178$ 